\title{
Energy-dispersed ions in the plasma sheet boundary layer and associated phenomena: Ion heating, electron acceleration, Alfvén waves, broadband waves, perpendicular electric field spikes, and auroral emissions
}

\author{
A. Keiling ${ }^{1}$, G. K. Parks ${ }^{1}$, H. Rème ${ }^{2}$, I. Dandouras ${ }^{2}$, M. Wilber ${ }^{1}$, L. Kistler ${ }^{3}$, C. Owen ${ }^{4}$, A. N. Fazakerley ${ }^{4}$, E. Lucek ${ }^{5}$, \\ M. Maksimovic ${ }^{6}$, and N. Cornilleau-Wehrlin ${ }^{7}$ \\ ${ }^{1}$ Space Sciences Laboratory, 7 Gauss Way, UC Berkeley, USA \\ ${ }^{2}$ Centre d'Etude Spatiale des Rayonnements, 9 ave de Colonel Roche, Toulouse, France \\ ${ }^{3}$ University of New Hampshire, 39 College Road, Durham, USA \\ ${ }^{4}$ Mullard Space Science Lab, Holmbury St. Mary, Dorking, UK \\ ${ }^{5}$ Space and Atmospheric Physics, Imperial College, London, SW7 2B2, UK \\ ${ }^{6}$ LESIA, Observatoire de Paris, Meudon, France \\ ${ }^{7}$ CETP, 10/12 Ave de l'Europe, Velizy, France
}

Received: 30 November 2005 - Revised: 8 June 2006 - Accepted: 29 August 2006 - Published: 20 October 2006

\begin{abstract}
Recent Cluster studies reported properties of multiple energy-dispersed ion structures in the plasma sheet boundary layer (PSBL) that showed substructure with several well separated ion beamlets, covering energies from $3 \mathrm{keV}$ up to $100 \mathrm{keV}$ (Keiling et al., 2004a, b). Here we report observations from two PSBL crossings, which show a number of identified one-to-one correlations between this beamlet substructure and several plasma-field characteristics: (a) bimodal ion conics $(<1 \mathrm{keV})$, (b) field-aligned electron flow $(<1 \mathrm{keV})$, (c) perpendicular electric field spikes ( $\sim 20 \mathrm{mV} / \mathrm{m})$, (d) broadband electrostatic ELF wave packets $(<12.5 \mathrm{~Hz})$, and (e) enhanced broadband electromagnetic waves $(<4 \mathrm{kHz})$. The one-to-one correlations strongly suggest that these phenomena were energetically driven by the ion beamlets, also noting that the energy flux of the ion beamlets was 1-2 orders of magnitude larger than, for example, the energy flux of the ion outflow. In addition, several more loosely associated correspondences were observed within the extended region containing the beamlets: (f) electrostatic waves (BEN) (up to $4 \mathrm{kHz}$ ), (g) traveling and standing ULF Alfvén waves, (h) field-aligned currents (FAC), and (i) auroral emissions on conjugate magnetic field lines. Possible generation scenarios for these phenomena are discussed. In conclusion, it is argued that the free energy of magnetotail ion beamlets drove a variety of phenomena and that the spatial fine structure of the beamlets dictated the loca-
\end{abstract}

Correspondence to: A. Keiling

(keiling@ssl.berkeley.edu) tions of where some of these phenomena occurred. This emphasizes the notion that PSBL ion beams are important for magnetosphere-ionosphere coupling. However, it is also shown that the dissipation of electromagnetic energy flux (at altitudes below Cluster) of the simultaneously occurring Alfvén waves and FAC was larger (FAC being the largest) than the dissipation of beam kinetic energy flux, and thus these two energy carriers contributed more to the energy transport on PSBL field lines from the distant magnetotail to the ionosphere than the ion beams.

Keywords. Ionosphere (Wave-particle interactions) - Magnetospheric physics (Auroral phenomena; Magnetosphereionosphere interactions)

\section{Introduction}

Ion beams are a characteristic feature of the PSBL during all levels of geomagnetic activity (Lui et al., 1978). Many studies have characterized their properties from low altitudes out to the distant magnetotail (e.g., Forbes et al., 1981; Eastman et al., 1984; Parks et al., 1984, 1998; Takahashi and Hones, 1988; Zelenyi et al., 1990; Bosqued et al., 1993; Hirahara et al., 1997; Sauvaud et al., 1999; Sergeev et al., 2000; Lennartsson et al., 2001; Grigorenko et al., 2002; Kazama and Mukai, 2003). To explain their existence, various processes have been proposed for the energization of ions in the

Published by Copernicus GmbH on behalf of the European Geosciences Union. 
(a) SC 1: Outbound crossing
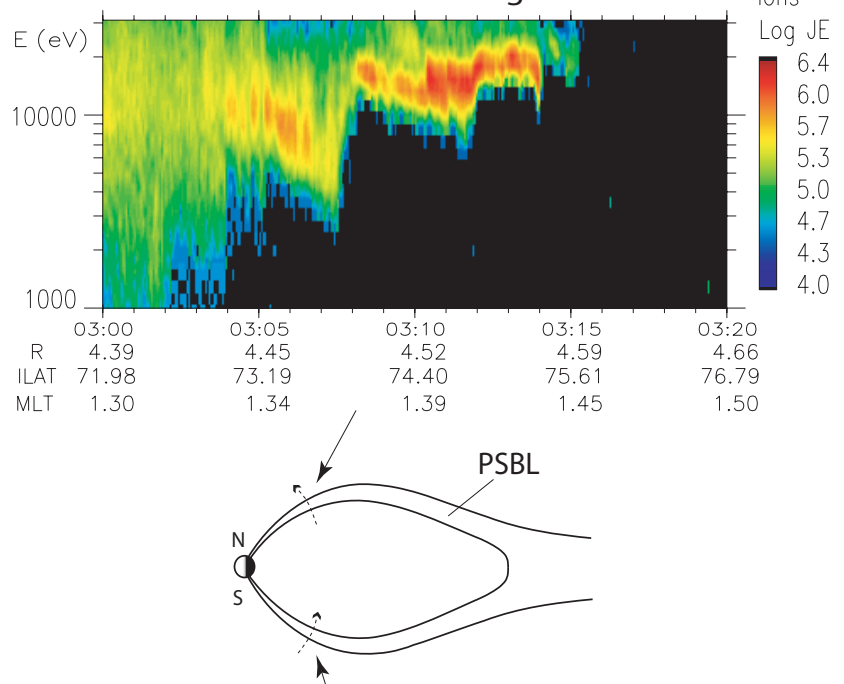

(b) SC 1: Inbound crossing

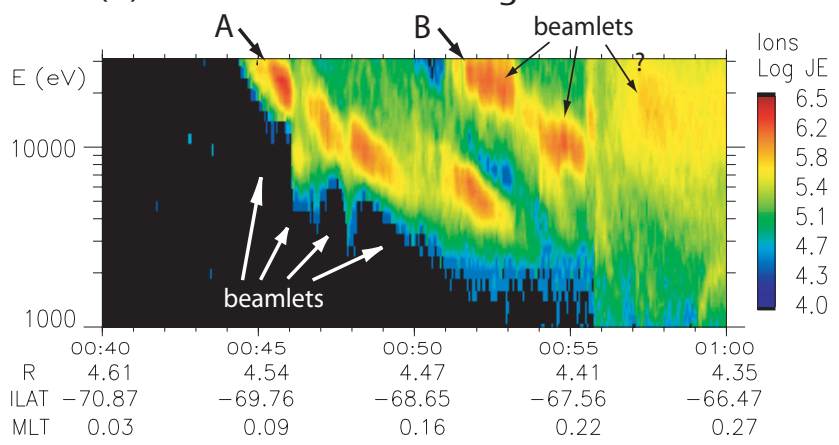

Fig. 1. Cluster crossings of the PSBL in both hemispheres on 14 February 2001, showing ion energy-time spectrograms for (a) the outbound crossing and (b) the inbound crossing (adapted from Keiling et al., 2004b).

magnetotail (e.g., Lyons and Speiser, 1982; Hasegawa, 1987; Schindler and Birn, 1987; Wygant et al., 2005). It is possible and even likely that different types of ion beams are generated by different mechanisms under different conditions. For example, it was found that the active and quiet magnetotail can lead to ion beams with different properties giving justification for the existence of several energization mechanisms (Sauvaud and Kovrazhkin, 2004).

In spite of ample observations, little has been conclusively confirmed about the impact of PSBL ion beams on auroral and magnetotail dynamics. The free energy carried in ion beams is clearly a potential source for driving other phenomena in the PSBL. Two general beliefs are that ion beams contribute to creating the aurora (e.g., Kan and Akasofu, 1976; Lyons and Evans, 1984) and to the formation of the central plasma sheet (CPS) (e.g., Lyons and Speiser, 1982). Early studies also found that ion flows in the PSBL simultaneously occur with various other field and plasma signatures (e.g., Parks et al., 1984; Eastman et al., 1984). In more recent studies, correlations between ion flows and other phenomena were further established. For example, energy-dispersed ions in the magnetotail have been directly associated with ionospheric and plasma sheet (PS) activities (Sauvaud et al., 1999; Sergeev et al., 2000; Kazama and Mukai, 2003). Some observational evidence has been reported that ion beams could lead to broadband electrostatic noise (BEN) (e.g., Gurnet et al., 1976; Grabbe and Eastman, 1984) via beam-related instabilities. Magnetotail ion beams have been invoked for the acceleration and heating of ionospheric ion outflow to form ion beams and conics, respectively (Alfvén and Fälthammar, 1963; Schriver et al., 2003; Lennartsson, 2003; Janhunen et al., 2003). Furthermore, Elphinstone et al. (1995) associated velocity-dispersed ion structures (VDIS) with the double oval, and Janhunen et al. (2003) suggested that the free energy associated with ion shell distributions in the PSBL could lead in a sequence of events to the acceleration of auroral electrons causing auroral arcs.

In this paper, we describe observations of phenomena which are associated with energy-dispersed ion structures in the PSBL for two events using a comprehensive set of instruments onboard Cluster. Both events have previously been investigated with regard to properties and generation mechanism of the dispersed ion structures (Keiling et al., 2004a, b). This new investigation will assess the impact of dispersed ion structures on their plasma environment and, in general, on magnetosphere-ionosphere (M-I) coupling. One of the chosen events is one of the most complex ones found in the three-year Cluster data base (2001 to 2003). On 14 February 2001 multiple energy-dispersed ion structures were recorded in the PSBL by the Cluster spacecraft while on an inbound crossing (Fig. 1b). At about 00:45 UT (00:10 MLT, 4.5 Re), Cluster 1 crossed the lobe-PSBL interface first followed by Cluster 3 (shown later). The ion data of Cluster 1 reveal four smaller-scale structures (called beamlets) which are grouped such as to resemble an extended dispersed ion structure (A); the second ion structure (B) contains two distinct beamlets. At 00:57 UT (arrow with question mark) a fainter ion structure is apparent but it is unclear whether it is a beamlet structure. Although this event is complex, its fine structure is very clear and shows well separated ion beamlets, allowing us in this study to less ambiguously identify relationships between ion beamlets and other phenomena, because we not only have one dispersed ion beam but several ion beamlets during one crossing, each of which can be compared to other local phenomena. These favorable circumstances warrant this comprehensive case study.

About two hours after Cluster crossed the PSBL in the Southern Hemisphere, it crossed the opposite (northern) boundary of the PS at about the same radial distance and local time but this time during an outbound motion of the spacecraft (Fig. 1a). Although the energetic ion signatures in the E-t spectrogram appear at first glance to be very different 
(best seen by comparing the dispersion slopes of the ions) from the inbound event (Southern Hemisphere), we proposed that in fact the same injection scenario operated during both inbound and outbound crossings creating the dispersion signatures (Keiling et al., 2004b). Consequently, one might expect that the impact of the dispersed ions on their plasma environment should be the same for both events, in spite of their differences in appearance in the E-t spectrograms, and we will show evidence for this.

The most significant result reported here will be to establish convincing one-to-one correlations among particle and field signatures which will lead us to propose a scenario in which the magnetotail ion beamlets are the driver of ion conics, perpendicular electric field spikes, field-aligned electron energization, broadband electrostatic waves, and electromagnetic waves. Furthermore, it will be proposed that the ion beams can be the driver of Alfvén waves and indirectly auroral emissions (via these Alfvén waves). In this study we will not, however, discuss the properties of the ion beamlets themselves and their possible generation mechanism in the far-tail but refer the reader to Keiling et al. (2004a, b) where properties such as energy range, pitch angle, composition, dispersion slopes and possible injection scenarios are discussed in detail. We also refer the reader to the simulation study by Ashour-Abdalla et al. (2005), who investigated one of the two events to identify the source region of these ion beamlets but came to an alternative interpretation.

\section{Data sources}

The observations presented here are from the Cluster spacecraft which are placed in a 57-h orbit with perigee and apogee of 4 and $19.6 R_{E}$ geocentric distance, respectively (Escoubet et al., 1997). Data used in this study come from the ion instrument (CIS), the electron instrument (PEACE), the electric field instrument (EFW), the plasma wave instrument (STAFF), and the Fluxgate magnetometer (FGM). In addition to the Cluster data, we utilized ground magnetic field data from the International Monitor for Auroral Geomagnetic Effects (IMAGE).

\section{First event: inbound crossing of the PSBL}

During the inbound crossing of the PSBL on 14 February 2001 at about 00:45 UT the substorm recovery phase prevailed according to ground magnetometer data from the IMAGE network (not shown). In this section we will show the following particle and field signatures occurring during this inbound crossing: field-aligned currents (FAC), heated ion outflows, accelerated electrons, broadband electrostatic and electromagnetic waves, electric field spikes, DC electric field, Alfvén waves, and energy flux calculations of the various magnetotail energy carriers. We will mostly describe observations from Cluster 1 but will point out differences between Cluster 1 and Cluster 3. First, we present a brief overview of this crossing including the tail lobe and the CPS to emphasize features that are unique to the PSBL.

\subsection{Plasma regions}

The Cluster spacecraft crossed several plasma regions during this event which can be identified in the energetic ion data $(>1 \mathrm{keV})$ (Figs. 2a and g), the low-energy ion data $(<3 \mathrm{keV})$ (Figs. $2 \mathrm{~b}$ and h), the electron data (Figs. $2 \mathrm{c}$ and i), the magnetic field (Figs. 2d and j) and electric field (Figs. 2e and k) data, and plasma wave data (Figs. $2 \mathrm{f}$ and 1 ).

Figure 2a shows the Cluster 1 encounter (first dashed line from left) with the magnetotail ion beamlets as was already shown in Fig. 1b. The beamlet-carrying region lasted until 00:56 UT (second dashed line) and is denoted the PSBL. Cluster 3 encountered the first ion beamlet about $1.5 \mathrm{~min}$ after Cluster 1 (separated by $\sim 500 \mathrm{~km}$ in the direction of motion and by $\sim 100 \mathrm{~km}$ in the azimuthal direction) but recorded a very different beamlet structure in the high energy ion data (Fig. 2g). Three larger scale structures (A, B, and C) were recorded of which $\mathrm{A}$ and $\mathrm{B}$ also show substructure in the form of beamlets but their separation is not as clear as it was for Cluster 1 (cf. Keiling et al., 2004a). This has direct consequences for establishing one-to-one correlations between ion beamlets and other phenomena. The region to the left of the PSBL is devoid of high energy ions and is the tail lobe. Following the PSBL to the right is a more structureless, thermalized ion population, which corresponds to the CPS (except of the faint structure at $\sim 00: 57$ UT indicated in Fig. 1b).

The three regions, identified on the basis of high energy ion data $(>1 \mathrm{keV})$, can also clearly be seen in the low-energy ion data (Figs. 2b and h). From the left, Cluster 1 first encountered outflowing ions with energies $<100 \mathrm{eV}$ in the tail lobe. Later we will show that this is $\mathrm{O}+$. No $\mathrm{O}+$ was recorded by Cluster 3. This region is followed by a different type of ion outflow covering the energy range up to about $1 \mathrm{keV}$ (starting at the first dashed line from the left). At the second dashed line a sharp transition occurs to a third kind of ion outflow, namely inverted ion V's with energies of $0.3-3 \mathrm{keV}$.

The electron data also show characteristic signatures in each region (Figs. 2c and i). First, to the left, polar rain is present which is the strongest evidence for the tail lobe. From 00:42 UT (Cluster 1) and 00:44 UT (Cluster 3) onward there is a gradual increase in electron energy up to several $\mathrm{keV}$. The region of $\mathrm{keV}$ electrons (between both dashed lines) also coincides with the presence of lower energy electrons $(<1 \mathrm{keV})$ which are intense and very structured. This structured signature is similar to the low energy ions (see Sect. 3.4 for more detail on this similarity). At the second dashed line, coinciding with the onset of the CPS as determined from the ions, the electron peak energy $(>10 \mathrm{keV})$ is raised on Cluster 1 but not on Cluster 3 , and the low energy electrons $(<1 \mathrm{keV})$ show a drop-out. 

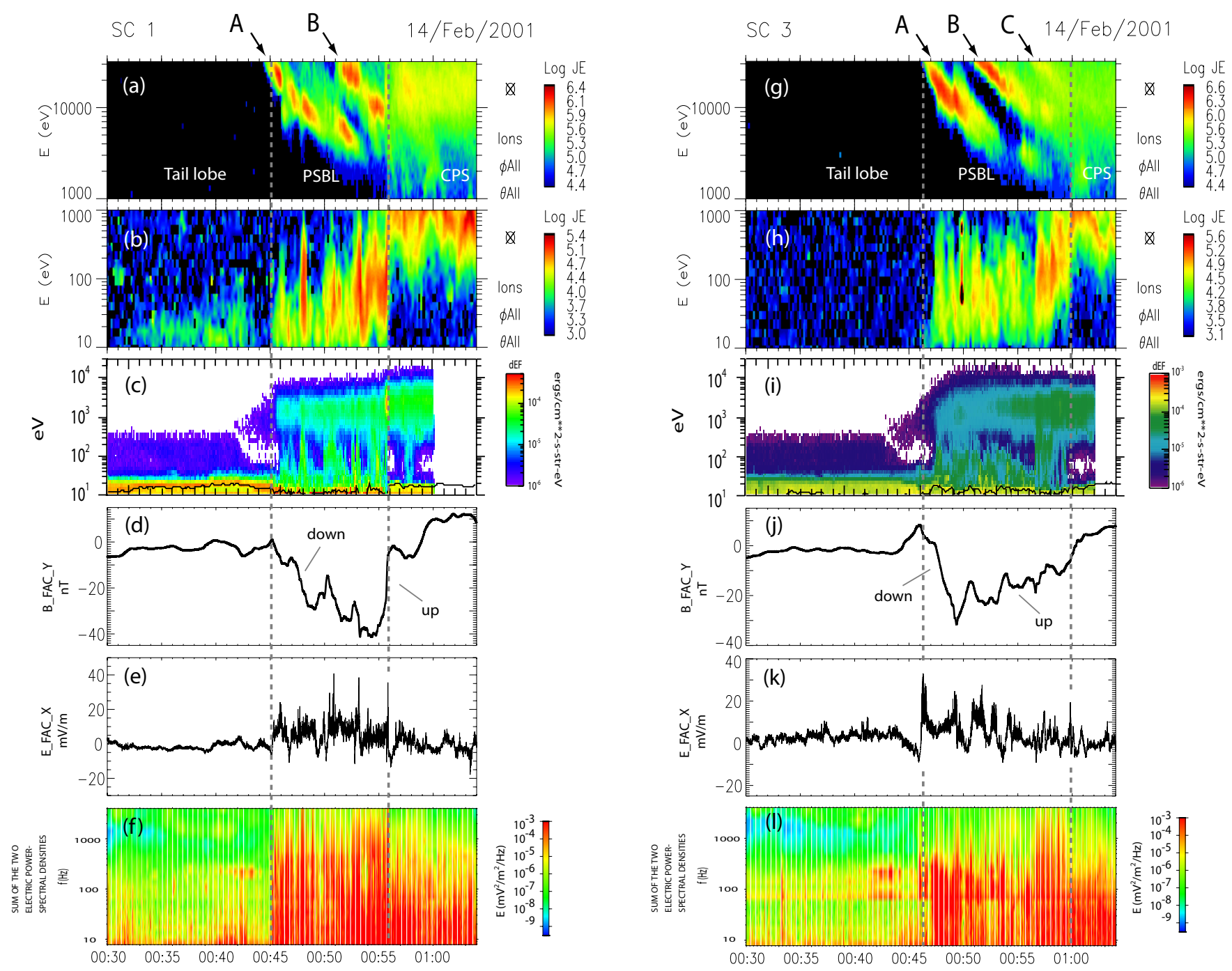

Fig. 2. Particle and field data during the lobe-plasma sheet crossing on 14 February 2001. (a-f) are for Cluster 1 . (a and b) Energytime spectrogram of all ions with $>1 \mathrm{keV}$ and with $<3 \mathrm{keV}$. (c) Energy-time spectrogram of electrons. (d). The azimuthal component of the magnetic field in field-aligned coordinates (4-s spin modulation removed). (e) Electric field component in $\mathrm{x}$ direction (field-aligned coordinates). (f) Sum of the two electric power spectral densities. (g-l) The same as (a-f) but for Cluster 3.

The plasma sheet (PSBL and CPS) was accompanied by field-aligned currents (FACs) which are inferred from the azimuthal magnetic field component (model subtracted) (Figs. 2d and j). On entering the ion PSBL, Cluster 1 recorded a downward FAC, followed by an upward FAC at approximately the time of entering the CPS. This is in contrast to Cluster 3, which recorded both downward and upward currents inside the PSBL. The significance of this difference is topic of Sect. 3.2. Note that the smaller-scale fluctuations superposed on the large-scale FAC in the beamletcarrying region for both spacecraft are due to Alfvén waves which will be further discussed in Sect. 3.5.

The division in different regions is also apparent in the electric field. On entering the PSBL, the fluctuations in the electric field increased (Figs. 2e and k), and on leav- ing the PSBL the fluctuation reduced significantly. Finally, we note that broadband electrostatic waves were enhanced and reached higher frequencies (up to $4 \mathrm{kHz}$ ) in the PSBL (Figs. 2f and 1).

\subsection{Field-aligned currents}

In Sect. 3.1 it was pointed out that upward and downward FACs were crossed by Cluster 1 and 3. The main difference between both spacecraft was the current flow direction change recorded by Cluster 3 inside the PSBL (Fig. $2 \mathrm{j}$ at $\sim 00: 50$ UT). This shows that the PSBL is not uniquely defined by the current flow direction. Consequently, all ion beamlets are found in the downward current region for Cluster 1 (cf. Figs. 2a and d), whereas for Cluster 3 (cf. Figs. 2g 
and j) they are found in both downward and upward currents. This difference can be explained by separating injected ion beamlets from bouncing echoes of these injections. It was recently argued by Keiling et al. (2004a, 2005a) that the beamlets of structure A (Figs. 2a and g) are separate, individual injections occurring in the distant tail, whereas the beamlets of structures $\mathrm{B}$ and $\mathrm{C}$ are not new injections but bouncing echoes of A. This distinction organizes the injected beamlets according to the current flow direction, namely all of the injected beamlets (structures A) are found in downward currents for both Cluster 1 and 3. This suggests that the injection region of the ions in the distant tail is a region of downward current.

On the other hand, the echo beamlets (structures B and C) can be found in both downward (Cluster 1) and upward currents (Cluster 3), suggesting that the echoes are independent of the current direction. Note that for Cluster 1, one echo beamlet of structure B overlaps with an injected ion beamlet of structure A at $\sim 00: 52$ UT. If the downward current is indeed of importance for the acceleration and injection of magnetotail ion beamlets, it is not inconsistent that the echo beamlets occur in upward FAC regions as well and this could be taken as additional evidence that these ion structures are echoes rather than new injections. The FAC flow directions with respect to the other plasma and field signatures are also of importance for establishing cause-effect relationships among various phenomena as will be described later.

The large-scale currents in the PSBL are associated with large-scale convective electric fields (see Sect. 3.5) and carry Poynting flux toward the ionosphere. This Poynting flux is shown in Sect. 3.7 and compared to the energy flux of other energy carriers in the PSBL such as ion flow and Alfvén waves.

\subsection{Ion outflow}

In Sect. 3.1 it was shown that the ion outflows could be divided into three regions on the basis of the variations of the ions' energy range. We now show for Cluster 1 additional ion composition and pitch angle data for each region which will reinforce the fact that the outflow in each region is distinct. Figures $3 \mathrm{a}$ and $\mathrm{b}$ are for reference showing the ion beamlets and the ion outflow (not mass-resolved from HIA). Figures $3 \mathrm{c}$ and d show mass-resolved E-t spectrograms of $\mathrm{H}+$ and $\mathrm{O}+$ (from CODIF) for energies $<3 \mathrm{keV}$. Figures $3 \mathrm{e}-\mathrm{g}$ show, respectively, pitch-angle spectrograms for non-mass-resolved ions, $\mathrm{H}+$, and $\mathrm{O}+$ for the same energy range $(<3 \mathrm{keV})$ as used in the corresponding E-t spectrograms. The horizontal black lines in Fig. 3e are visual aids to emphasize the different pitch angle ranges in each region.

The ion outflow in the tail lobe is identified as purely $\mathrm{O}+$ (Figs. 3b-d) with peak energy and pitch angle range of $<100 \mathrm{eV}$ and $<50^{\circ}$, respectively. The ion outflow in the PSBL contains both $\mathrm{H}+$ and $\mathrm{O}+$. Energies and pitch angle ranges for $\mathrm{H}+$ are $10-800 \mathrm{eV}$ and $<90^{\circ}$, respectively. $\mathrm{O}+$, on the other hand, has the same pitch angle range but is less energetic than $\mathrm{H}+$. In contrast, the ion outflows of $\mathrm{H}+$ and $\mathrm{O}+$ in the CPS are more energetic and the pitch angle range of $<30^{\circ}$ is significantly narrower compared to the PSBL ions. Two instrumental considerations are noted here. First, the ion energy measurements are with respect to the floating spacecraft potential. Since this potential is between 10 to $20 \mathrm{eV}$ in the PSBL (as determined from the electric field instrument EFW, not shown), the energy values given above need be corrected upward. Second, to verify that $\mathrm{O}+$ is not an artifact (i.e., spillover from $\mathrm{H}+$ ) in the CODIF measurements, we additionally verified the presence of $\mathrm{O}+$ by analyzing time-of-flight histograms (not shown here). A word of caution is that these histograms required time intervals of several minutes to accumulate enough data points to be certain that the noise level was exceeded. Thus, individual O+ structures could not always be independently verified. The analysis that follows will thus focus on $\mathrm{H}+$.

The CPS ion $(\mathrm{H}+)$ outflow shows inverted $\mathrm{V}$ structures (see arrows in Fig. 3b) which have typical beam distributions (Figs. 4e and f). The distribution functions of the $\mathrm{H}+$ outflows in the PSBL, on the other hand, are conical with varying cone angles, showing both parallel and perpendicular acceleration (Figs. 4a-d). The distribution in Fig. 4c is bowl-shaped (white dashed line) and is very similar to those discussed in Kumplar et al. (1984), where it is argued that the ions undergo at least two acceleration mechanisms, one field-aligned and the other perpendicular to the background magnetic field. The variation in cone angles indicates that the transverse energization occurred at various altitudes from near the spacecraft (Fig. 4a) to $\sim 6000 \mathrm{~km}$ below the spacecraft (cone angle of $90^{\circ}-$ Fig. $4 \mathrm{~b}$ ). The latter estimate is based on the assumption of constant energy and constant magnetic moment during travel. Since some conics were energized well below the spacecraft, energy dispersion over the energy range of the conics might be expected. However, no dispersion was present within the limit of the detector's time resolution. One possible explanation is that the heating region was moving with the large-scale $\boldsymbol{E} \times \boldsymbol{B}$ drift (see Sect. 5.1 for further discussion).

Because of their different properties (note the abrupt change at $\sim 00: 56 \mathrm{UT}$ ), it is to be expected that the energization of the ion outflows in the PSBL and those in the CPS are the result of different processes. It was noted above that there is a clear distinction of down- and upward current in the PSBL and CPS, respectively, for the crossing recorded by Cluster 1 . This, however, was not the case for the crossing recorded by Cluster 3 where both down- and upward currents existed in the PSBL. Since ion outflow existed throughout the PSBL crossed by both spacecraft, we rule out that the FACs were the driver of the ion outflows in the PSBL. On the other hand, the large-scale FAC in the CPS are upward for both Cluster 1 and 3. Field-aligned potential drops created in upward FAC are probably the cause for the inverted V ion beams in the CPS (Carlson et al., 1998). The 


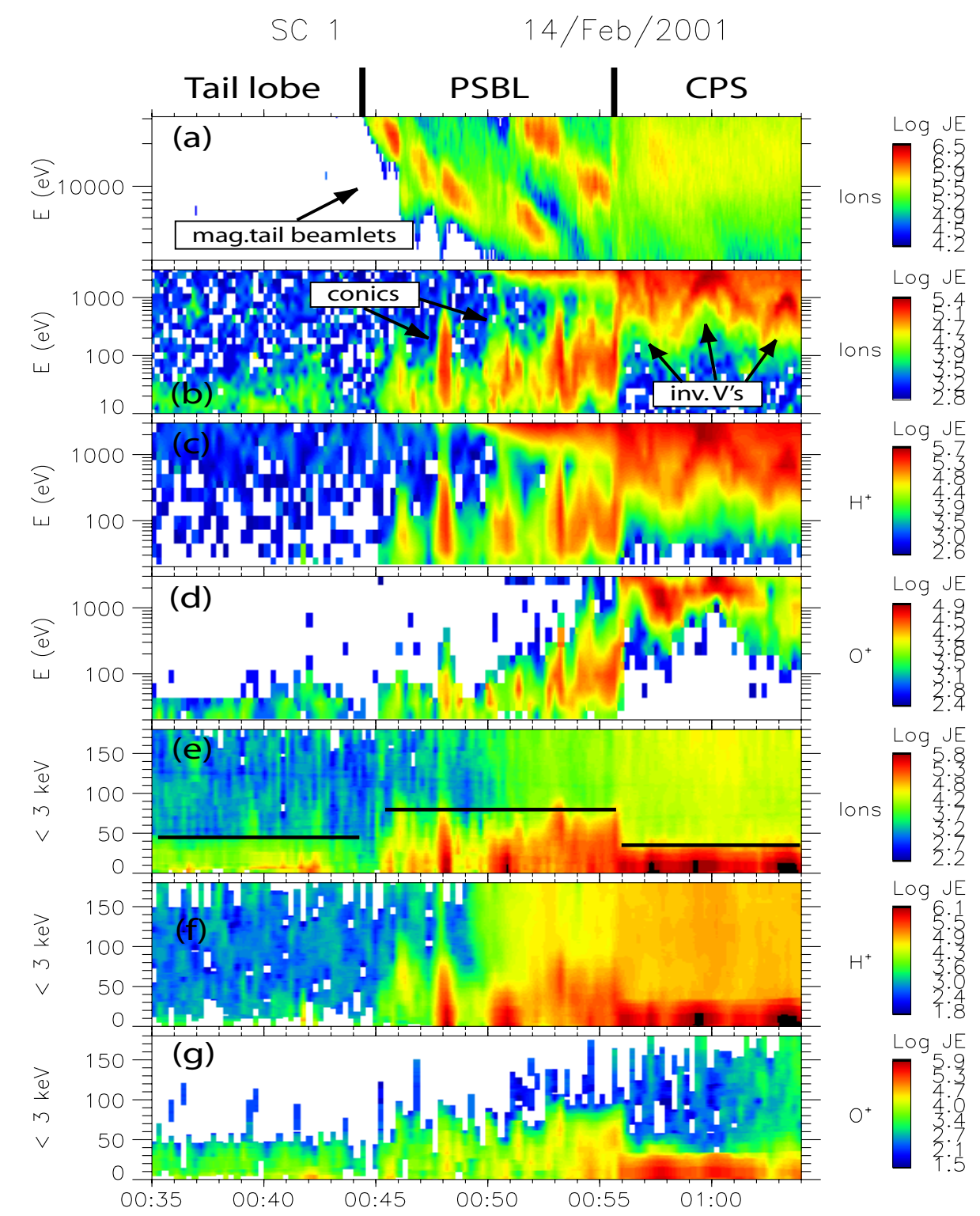

Fig. 3. Composition and pitch angle data for Cluster 1 on 14 February 2001. Energy-time spectrograms of (a) all ions with $>3 \mathrm{keV}$, (b) all ions with $<3 \mathrm{keV}$, (c) $\mathrm{H}+$ with $<3 \mathrm{keV}$, and (d) $\mathrm{O}+$ with $<3 \mathrm{keV}$. (e-g) Pitch angle versus time spectrograms for all ions, $\mathrm{H}+$ and $\mathrm{O}+$, respectively.

most obvious difference between the ion conic region and the neighboring regions is the simultaneous presence of magnetotail ion beamlets in the ion conic region, which strongly suggests their importance for the energization of the ion outflows. Additional support for the scenario in which the magnetotail beamlets are responsible for the conic generation is a close spatial/temporal relationship between ion beamlets and individual ion outflow structures for Cluster 1 (dashed lines with arrows in Figs. 5a and b). The ion outflow structures show a similar periodicity as the magnetotail ion beamlets suggesting that the latter causes the former. Moreover, it is important to note that the ion outflow structures occurred at or in close vicinity to the "edges" of the magnetotail beamlets. Figure $5 \mathrm{~d}$ shows higher time resolution $(12 \mathrm{~s}$ versus $16 \mathrm{~s}$ in Fig. 5b) non-mass resolved ions (i.e., including both $\mathrm{H}+$ and $\mathrm{O}+$ which accounts for the differences to Fig. 5b) confirming this association with the "edges". Figure 5c shows particle flux rather than energy flux as in Fig. 5b. A comparison of these two figures shows that there is also ion outflow away from the beamlet edges but the most energetic outflow occurred close to the beamlet edges. If the beamlets (in particular their edges) are responsible for the ion heating, as will be argued in this report, it might be argued that the conics that were energized far below the spacecraft should be spatially 


\section{PSBL}

(a)

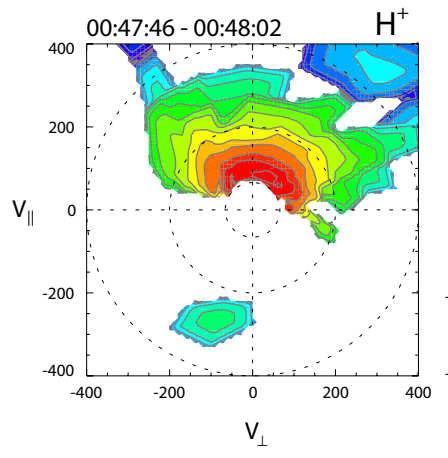

(b)

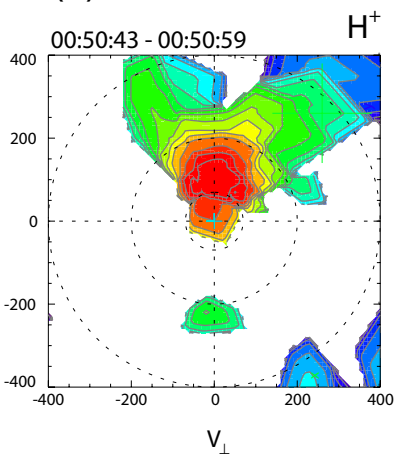

(c)

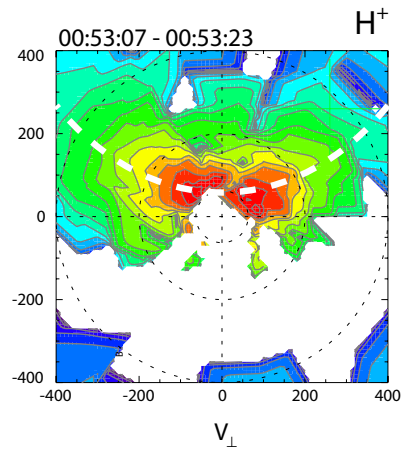

(d)

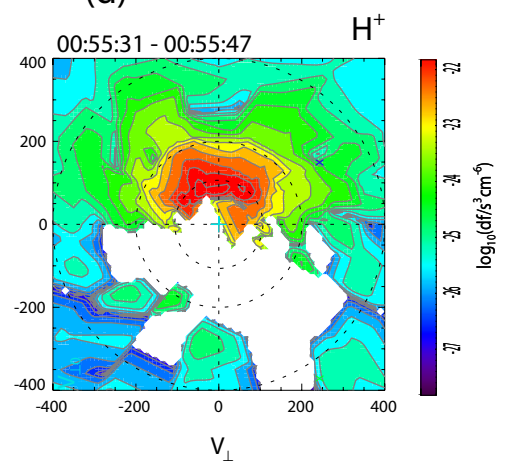

\section{CPS}

(e)

(f)
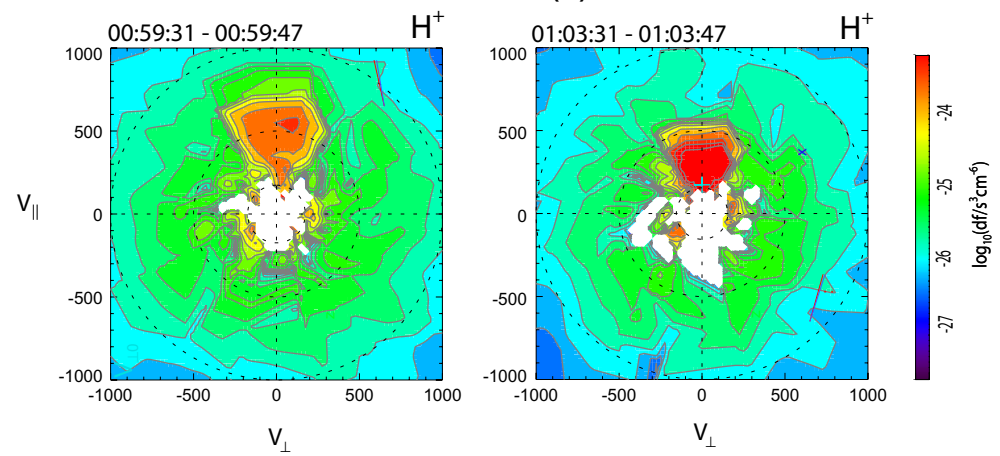

Fig. 4. Velocity space distribution functions of Cluster 1 for specific times during the plasma sheet crossing on 14 February 2001. (a-d) show ion conics in the PSBL, and (e) and (f) show ion beams in the CPS.

displaced from the ion beamlets because of the presence of large-scale convection during the time period the conics traveled from their source region to the spacecraft. This lack of significant displacement can be explained with the simultaneous $\boldsymbol{E} \times \boldsymbol{B}$ drift of the ion beamlets and the ion conics (see Sect. 5.1 for further discussion).

In Sect. 3.5, we will identify Alfvén waves collocated with the beamlet-carrying region which also needs to be considered as a driver of ion heating. However, we will argue against such a scenario (see Sect. 5.1).

For SC 3, ion outflows also occurred in the same region as the magnetotail beamlets. The energy range of this outflow is the same as observed by Cluster 1 . However, no convincing one-to-one correlation was apparent (see Figs. $2 \mathrm{~g}$ and $\mathrm{h}$ ). This could be explained with the lack of beamlet separations with clear edges during the Cluster 3 crossing. A second event will be presented later (Sect. 4) which will reinforce the importance of beamlet edges for the energization of ion conics. It is also noted that two outflow structures (between 00:47-00:50 UT in Fig. 2h) showed energy dispersion in the Cluster 3 data; all other structures showed no dispersion. For the moment, we hypothesize that these structures were cre- ated by a separate mechanism which will be further investigated elsewhere.

\subsection{Accelerated electrons}

The electrons $(<1 \mathrm{keV})$ show a structured signature in the beamlet-carrying region (PSBL) (Figs. 5e-g) which abruptly stops at 00:56 UT coinciding with the exit of the beamletcarrying region, which is also equivalent to leaving the ion conic outflow region. At about 00:57 UT electron flow was again recorded which coincides with more intense PS ions (Fig. 5a, arrow \#6). Although we did not classify this ion structure as a PSBL beamlet in the previous sections, it could be argued that it is a thermalizing remnant of a bouncing PSBL beamlet as discussed in Keiling et al. (2005a). No outflowing ion conic was associated with this ion structure.

The most interesting observation regarding the electrons is that at times of ion conic outflows (arrows labeled 1 through $5)$, the electron flow was more intense and reached higher peak energy values $(>100 \mathrm{eV})$ compared to the adjacent electrons. The energies were comparable to the ion outflow energies. The one-to-one correlation between ion conics and 


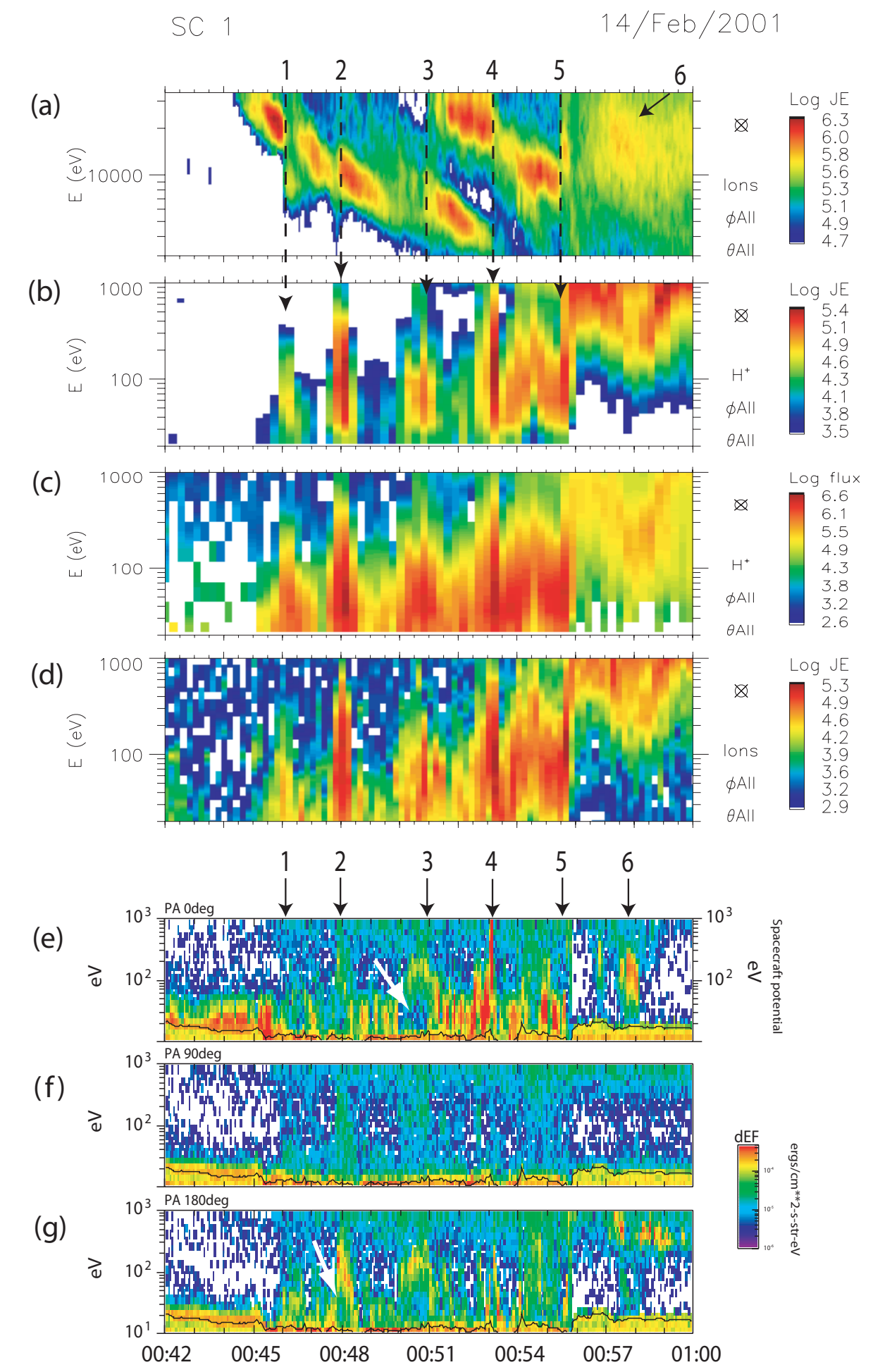

Fig. 5. Comparison of ion and electron signatures during the PSBL crossing on 14 February 2001 for Cluster 1 . The dashed lines with arrows in panel (a) show the correlation with ion beamlets and ion conics (a-d). Note that the color scale in (b) and (c) is energy flux and flux, respectively. Panel (d) shows non-mass resolved ion data which has a higher time resolution (4s) than the H+ data (12 s) in (b). The electron data are separated in (e) field-aligned, (f) perpendicular, and (g) anti-field aligned energy-time spectrograms. 
electron "bursts" is best seen for the structures labeled 2, 3, and 4 which also coincides with times that show the clearest beamlet separation. Furthermore, the electron bursts 2 and 3 do not show low energies $(<100 \mathrm{eV}$, white arrows in Figs. 5e and $g$ ) as in the neighboring regions suggesting that the electrons were simply raised in energy from their background values. These observations suggest that the mechanism that caused the heating of the ion conics was also directly or indirectly causing the electron acceleration. However, in contrast to the unidirectional ion outflow in this region, the electron flow direction is more complex; electrons are observed in all three angular sectors (panels $\mathrm{e}-\mathrm{g}$ ) in combinations of field-aligned, anti-aligned and perpendicular motion that depend upon time and/or location. For example, the electron bursts labeled 2 and 3 are seen at all pitch angles with the downward direction (panel g: PA 180 degree) being the most intense. Downgoing electrons suggest that electron acceleration processes also occurred above Cluster's altitude. Where down- and upgoing electrons occurred together, it is probable that the upgoing electrons were the reflected downgoing electrons; note that in these cases both upgoing and downgoing electrons had the same energies (e.g., burst \#3). Perpendicular flowing electrons are probably locally mirroring ones. Some electron structures (e.g., burst \#4) show mostly upward flow, suggesting that the electrons were accelerated below the spacecraft. Consequently, Cluster was immersed in a region which experienced field-aligned electron acceleration above and below the spacecraft.

In Sect. 3.3, we established a one-to-one correlation between magnetotail ion beamlets and ion conics, and thus we argued that the former provides the energy for the latter. The same can be suggested for the electrons, that is, the ion beamlets are causally related to the energization of the electrons. However, it is not necessary that both ion conic and electron energization occurred at the same location simultaneously, since we showed electrons that were energized above the spacecraft but were recorded together on the same field lines with ion conics that came from below the spacecraft (see, for example, the ion and electron structures labeled 2).

\subsection{Electric and magnetic field variations}

On crossing the PSBL, enhanced electric field activity persisted (Figs. 2e and k). Two expanded views of this crossing are shown in Figs. 6 and 7 for Cluster 1. The electric field shows several features which are superposed such as small-scale electric field spikes, higher frequency electrostatic fluctuations, the perturbation field of low-frequency Alfvén waves, and a DC electric field shift. Below we investigate each of these features. The magnetic field, on the other hand, shows low-frequency variations superposed on the large-scale FAC. These low-frequency variations are associated with the Alfvén waves. Higher frequency electromagnetic waves are also present. $\mathrm{E}$ and $\mathrm{B}$ are shown in fieldaligned coordinates, with $\mathrm{z}$ along $\mathrm{B}, \mathrm{y}$ westward, and $\mathrm{x}$ mak- ing a right-handed coordinate system (i.e., nearly radially inward).

\subsubsection{Electric field spikes}

Obvious features in the electric field inside the PSBL are isolated, larger amplitude spikes (arrows in Fig. 6b). These spikes have a periodicity of $1-3 \mathrm{~min}$ which is comparable to the periodicity of magnetotail ion beamlets. Moreover, the spikes are often found at the edges of individual beamlets (best seen for the largest spikes). This correlation is demonstrated in a different format in Fig. 6c, where both the density of the ion beamlets with energies $>3 \mathrm{keV}$ and a filtered $(>0.3 \mathrm{~Hz})$ component of the electric field are overlaid. (Note that the density plot is 12 -s time resolution whereas the E-t spectrogram is 4-s time resolution.) Individual larger spikes with amplitudes up to $20 \mathrm{mV} / \mathrm{m}$ are located at density gradients. This one-to-one correlation suggests that the spikes are causally related to the ion beamlets, in particular their edges or density gradients.

\subsubsection{Broadband waves}

In addition to large electric field spikes, extremely low frequency (ELF) electric field turbulence (Fig. 6c) as determined from EFW (Nyquist frequency of $12.5 \mathrm{~Hz}$ ) as well as broadband electrostatic noise (BEN) with frequency up to $4 \mathrm{kHz}$ as determined from the plasma wave instrument STAFF (Fig. 6d) occurred throughout the beamlet-carrying region. There are no corresponding magnetic field fluctuations (Fig. 6e), except at specific times (see below) when broadband electromagnetic waves were recorded by the wave instrument.

According to the EFW data, three broad regions of enhanced ELF electrostatic waves, indicated by horizontal bars in Fig. 6c, can tentatively be identified. The two regions to the left coincide with the magnetotail beamlets (bars in Fig. 6a), but the region to the right is located inside the CPS. Collocated with the ELF region in the CPS was a somewhat increased intensity in ion energy flux. This weak ion structure has already been pointed out in Sect. 3.4 where it was shown that it also coincided with enhanced electron flow. The regions of ELF waves show signatures of wave packets. Thus an obvious question is whether these wave packets are associated with other particle signatures. In Fig. $6 \mathrm{~g}$ magnetotail ion beamlets and wave packets are shown for a sub-interval with vertical dashed lines drawn above individual wave packets. Many of these lines line up with the start and end of individual beamlets or with smaller ion features. For example, the third wave packet (\#3) lines up with a faint beamlet feature that is located between two larger beamlets of structure A (cf. Fig. 2a). Between wave packets \#5 and \#6 and between \#9 and \#10, wave turbulence prevails without clear wave packets. These two periods correspond to beamlets that lasted somewhat longer (1-2 min). Wave packets, 


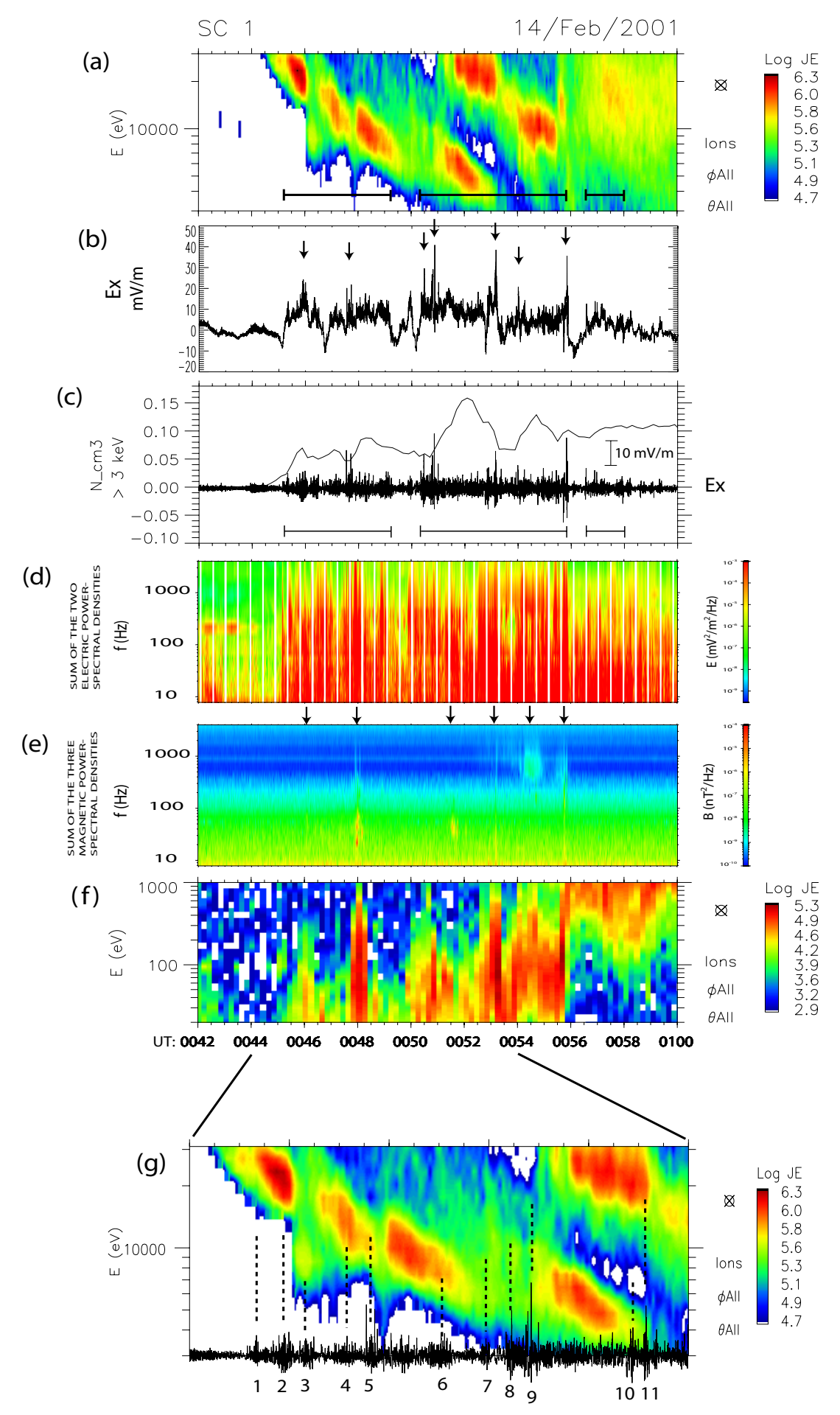

Fig. 6. Comparison of ion beamlets with electric field and wave data for the 14 February 2001 event. (a) E-t spectrogram of ion beamlets. (b) $\mathrm{E}_{x}$ component of the electric field (field-aligned coordinate system). (c) High-pass filtered electric field overlaid on the density of the ion beamlets $(>3 \mathrm{keV})$. (d, e) Electric and magnetic field power spectral densities (up to $4 \mathrm{kHz}$ ). (f) E-t spectrogram of ion conics for a comparison with the electric and magnetic field data. (g) Expanded view of the filtered electric field from panel (c) together with the ion beamlets from panel (a). 
spikes and beamlet gradients are possibly related which will be further discussed in Sect. 5.3.

On entering the PSBL, BEN with frequencies $>100 \mathrm{~Hz}$ were recorded which continued deep into the CPS. However, coinciding with the beamlet-carrying region (PSBL) the frequency reached values up to the limit of the instrument $(4 \mathrm{kHz})$. BEN was striated throughout the PSBL so that a simple and convincing one-to-one correlation with the wave packets of the ELF waves was not possible to establish. Similarly, it is more ambiguous as to whether there was a clear one-to-one correlation with ion conics (Fig. 6f). However, at specific times (arrows above panel e), broadband magnetic fields were recorded with corresponding broadband electric fields (panel d), thus showing the presence of electromagnetic waves. Interestingly, these waves were correlated with the edges of ion beamlets (Fig. 6a) (or, equivalently, outflowing ion conics), again suggesting a causal relationship.

\subsubsection{Alfvén waves}

The nature of the low-frequency fluctuations can be seen in the band-pass filtered (40-160 s) electric and magnetic field data (Figs. 7c-f). This filter range shows the lowest frequency Alfvén waves during this crossing but higher frequencies waves were also present. Figures $7 \mathrm{a}$ and $\mathrm{b}$ show E (unfiltered) and B (filtered: $>4 \mathrm{~s}$ ). To compare the phase relationship between E and B, Fig. 7e shows the electric field overlaid with the Hilbert-transformed magnetic field. The Hilbert transform shifts all frequency components by $90^{\circ}$ without changing their magnitudes. This technique has been demonstrated by Dubinin et al. (1990). It can be seen that both fields show similar waveforms with reduced phase shift during the time period indicated by the horizontal bar. Because B was phase-shifted by the Hilbert transform, this result indicates that the E and B fluctuations were partially standing Alfvén waves. We also plotted in the last panel $\mathrm{E}$ and $\mathrm{B}$ (without Hilbert transform) to show that these two wave forms do not match as well. It is noted that the E-to-B ratios are smaller than the local Alfvén speed; however, a deviation is to be expected for a mixture of traveling and standing Alfvén waves (Mallinckrodt and Carlson, 1978). We emphasize that the identification of traveling or standing waves in the PSBL depends on the chosen frequency range (Keiling et al., 2005b). In other frequencies ranges more traveling wave power was observed for this crossing.

After the region of Alfvén waves, a strong current was encountered ( 00:56 UT), and there $\mathrm{E}$ and $\mathrm{H}(\mathrm{B})$ are not in phase; instead $\mathrm{E}$ and $\mathrm{B}$ are in phase. Furthermore, the E-to-B ratio in this case is significantly smaller than the local Alfvén speed, indicating that this is a static current structure.

\subsubsection{DC electric field}

Finally, a DC electric field of $5-10 \mathrm{mV} / \mathrm{m}$ was present in the beamlet-carrying region (Fig. 6b). This DC field to-
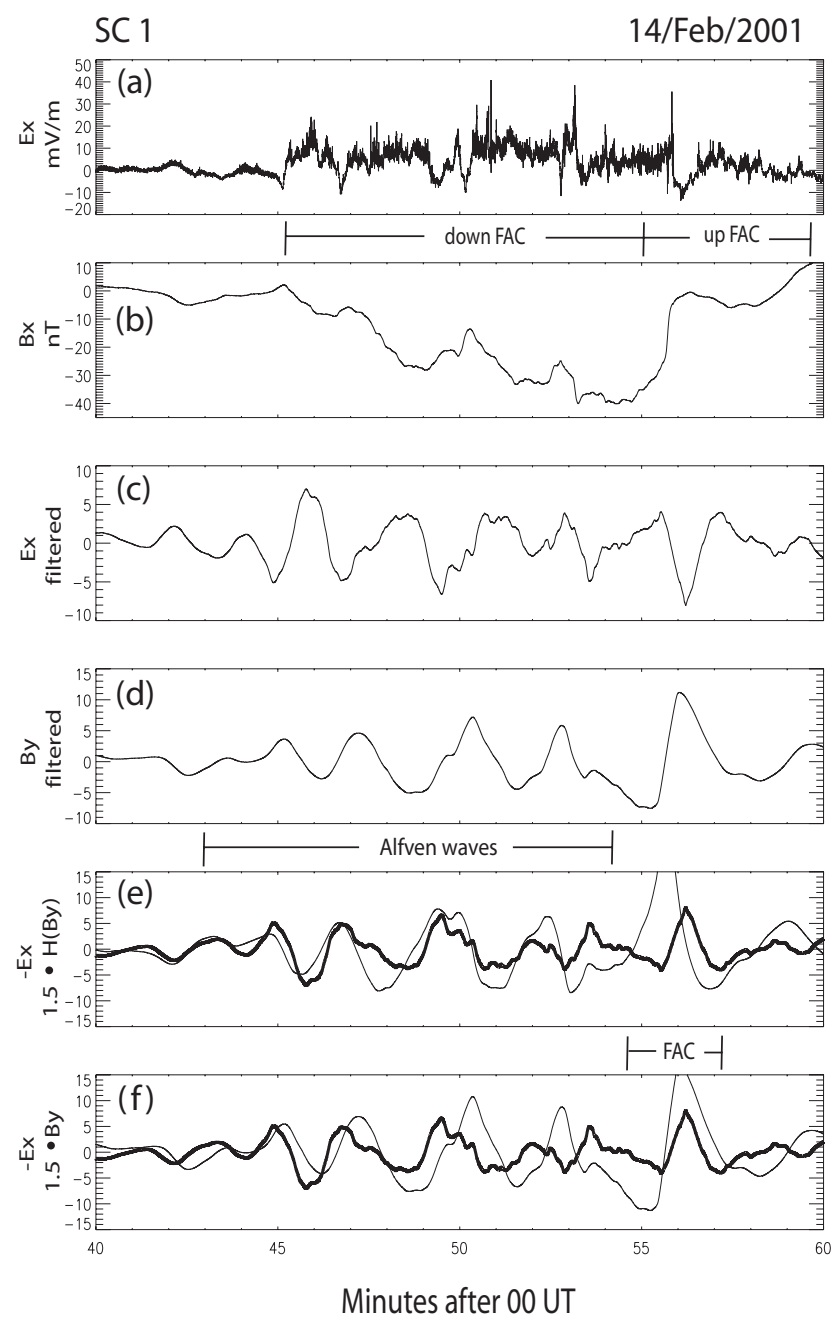

Fig. 7. Electric and magnetic field data during the PSBL crossing on 14 February 2001 for the analysis of the low-frequency variations. (a) Unfiltered and (b-f) filtered electric and magnetic fields. The field data are presented in field-aligned coordinates. Shown are the Alfvén wave components in the range from 40-160 s. The last two panels (e, f) show comparisons of E, B, and the Hilbert-transformed B. $90^{\circ}$ phase-shifts or lack thereof are indicators for standing and traveling Alfvén waves.

gether with the total magnetic field yield a large-scale convective plasma flow $\left((\boldsymbol{E} \times \boldsymbol{B}) / \mathrm{B}^{2}\right)$ of the order of $10 \mathrm{~km} / \mathrm{s}$ in the azimuthal direction (determined from EFW and FGM; not shown). This convective plasma flow is possibly associated with shear flow in the distant tail which mediates magnetic stress via FAC and DC electric field to the Cluster location.

Furthermore, the DC electric field and the magnetic field of the field-aligned current carry Poynting flux towards the ionosphere which is calculated and compared to other energy carriers in Sect. 3.7. 

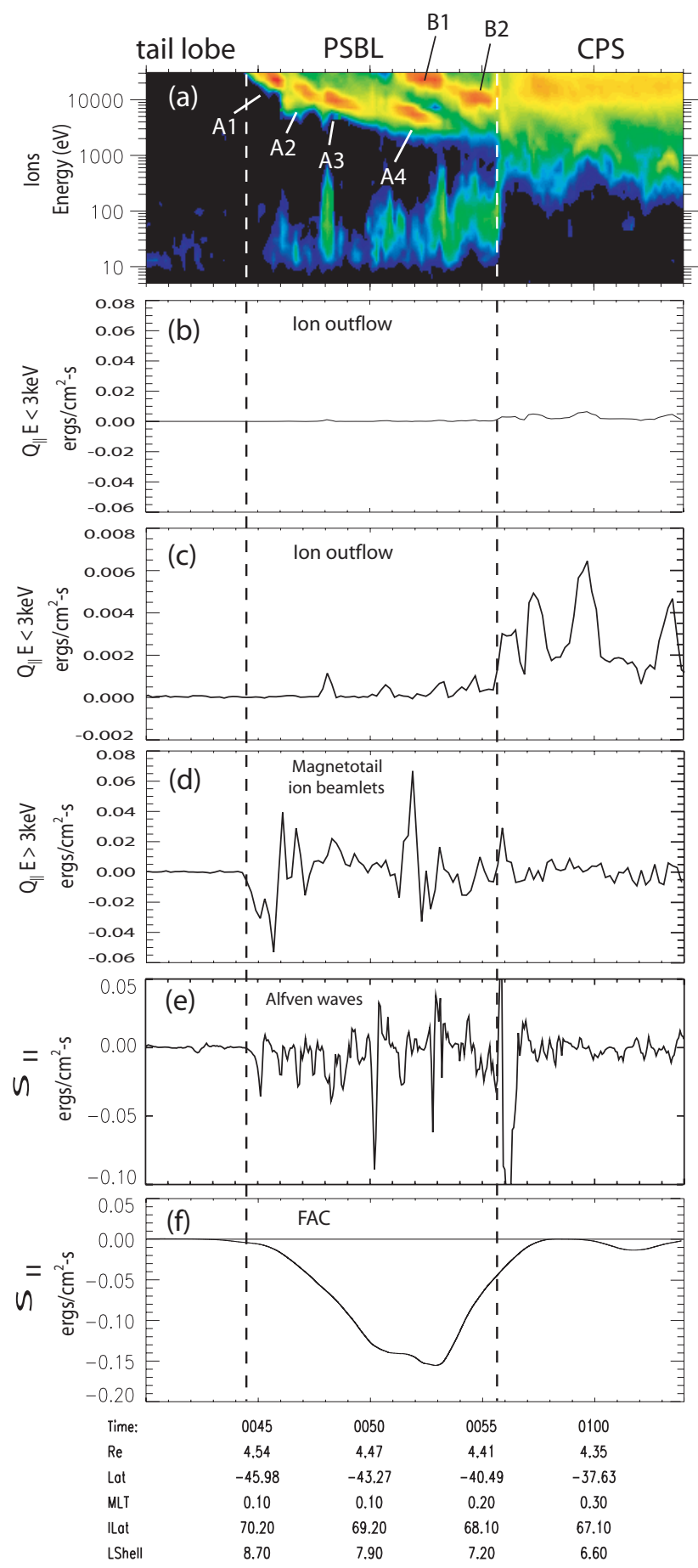

Fig. 8. Energy flux comparison of (b, c) ion outflow, (d) magnetotail ion beamlets, (e) Alfvén waves, and (f) FAC. (a) shows the E-t spectrogram of ions as a reference. Note that (b) and (c) show the same ion data $(<3 \mathrm{keV})$ but with different y scale. The vertical dashed lines bracket the PSBL.

\subsection{Auroral emissions}

At the time of the PSBL crossing on 14 February 2001, ultraviolet images from the IMAGE satellite show a double oval (as reported by Keiling et al., 2004a) which is a typical recovery phase signature (Elphinstone et al., 1995). Allowing for the mapping uncertainty, it is possible that the ion beamlets were conjugate to the poleward arc of the double oval, and that the ion beamlets were indirectly responsible for the auroral emissions. In particular, the question arises whether the sharp beamlet gradients that showed large perpendicular electric field spikes were conjugate to individual arcs. An alternative source for the poleward arcs of the double oval are the Alfvén waves that were simultaneously present. Both possibilities are discussed in Sect. 5.7.

\subsection{Energy flux}

In order to determine the energy source of the phenomena that have been presented in the previous sections, such as the ion heating and electron acceleration, broadband waves, and auroral luminosity, it is necessary to investigate the energy flux flowing towards Earth and crossing the region that was traversed by Cluster. Contributors to this energy flux are the magnetotail ion beamlets, FAC and the Alfvén waves. Figure 8 shows in the first panel the E-t spectrogram of the ions with individual beamlets being labeled. The following panels show the field-aligned energy flux associated with the ion outflow $(<3 \mathrm{keV})$ with two different scales (panel b has the same scale as panel $\mathrm{d}$ for better comparison), the magnetotail ion beamlets $(>3 \mathrm{keV})$, the Alfvén waves (filtered: $6 \mathrm{~s}, 180 \mathrm{~s}$ ), and the large-scale FAC (E and B were detrended with a 5-min running average to obtain the DC component). Once again there is a clear distinction in the three regions (tail lobes, PSBL, CPS) with respect to the energy flux. There is near zero energy flux in the tail lobe for all quantities. The energy flux of the ionospheric ion conics in the PSBL is about one order of magnitude smaller compared to the inverted V's in the CPS. For all magnetotail drivers (panels d, e, and f), the energy flux is largest in the PSBL. The energy flux of each driver is $1-2$ orders of magnitude larger than the ion conics energy flux, and, therefore, the energy flux of each driver is in principle sufficient to energize the ion conics.

It is noted that the energy fluxes of the magnetotail ion beamlets and the Alfvén waves show a tailward directed contribution. For Alfvén waves, this is due to reflected Alfvén wave components as shown in Sect. 3.5. The tailward energy flux for the ions is caused by temporal/spatial effects which can be seen in velocity space distributions (Fig. 9). Some distributions have already been reported in Keiling et al. (2004a). For example, Figs. 9b, e, and h show full shell distributions whereas Figs. 9a, c, and i show partially filled distributions. These variations reflect temporal/spatial effects. For example, the first three distributions (Figs. 9ac) show the changes associated with beamlet A1. The first 


\section{A1}

(a) $00: 45: 34.882$

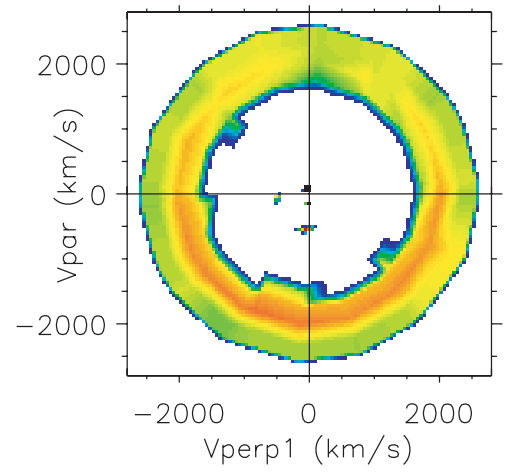

(b) $\quad 00: 45: 46.890$

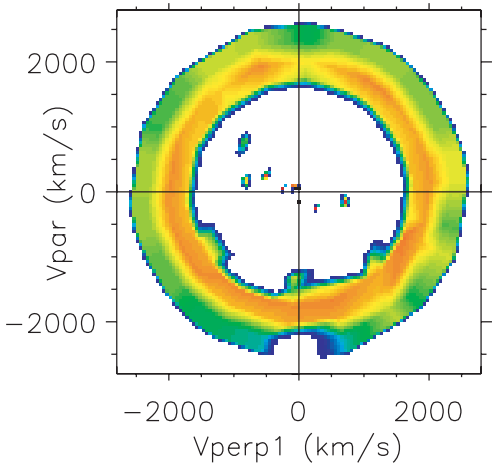

(c) $00: 45: 58.907$

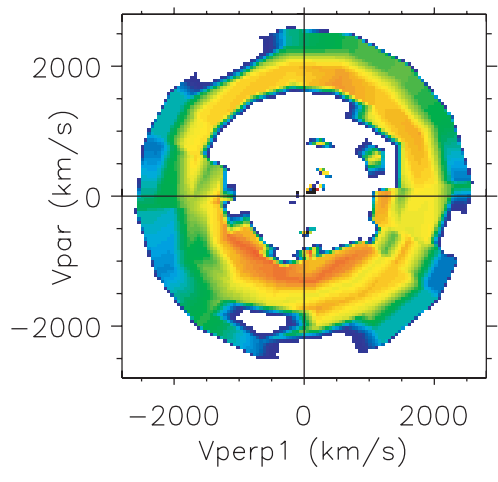

A2

(d) $00: 46: 58.936$

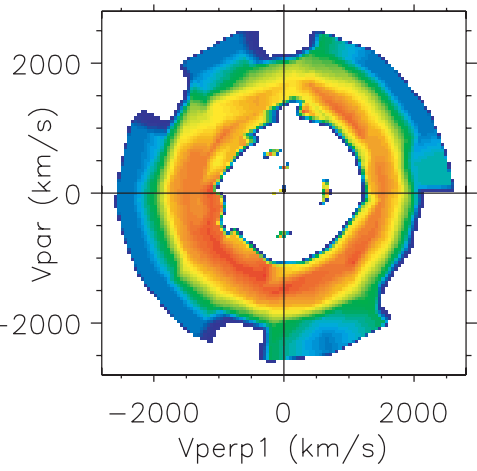

(e) $00: 47: 10.952$

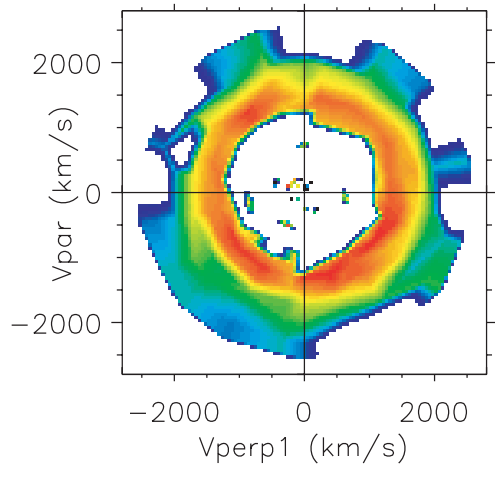

A3

(f) $00: 48: 10.997$

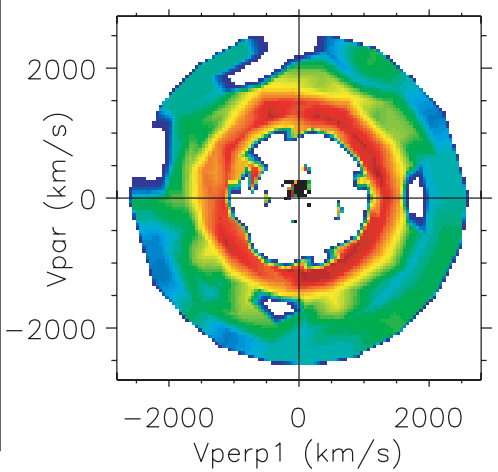

A4 and B1

(g) $00: 51: 47.155$

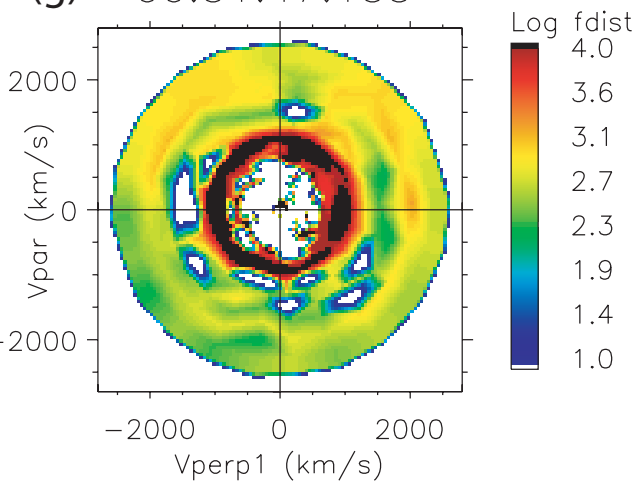

(h) $\quad 00: 52: 23.182$

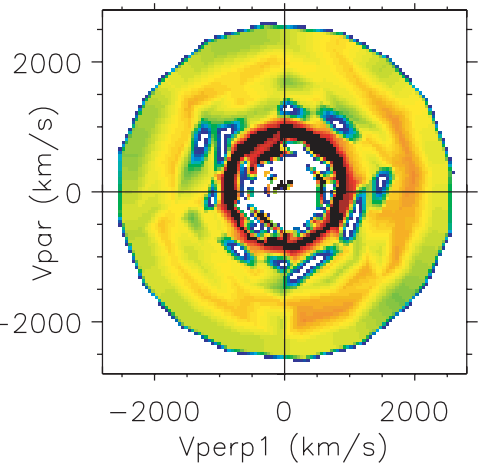

(i) $00: 52: 35.183$

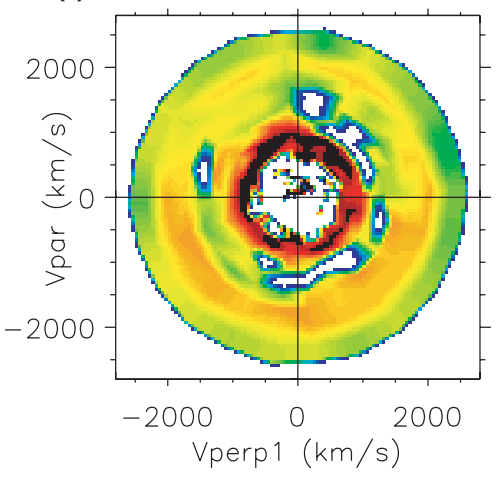

Fig. 9. Selected velocity space distribution functions (Cluster 1 - HIA) for various ion beamlets during the PSBL crossing on 14 February 2001. Negative (positive) velocities indicate downward (upward) flow directions.

encounter with A1 shows mostly downgoing ions (panel a) followed by downgoing and mirroring ions further into the PSBL (panel b). On leaving A1, mostly mirroring ions were recorded (panel c). Moreover, a closer inspection reveals that the peak upward velocity in panel (b) is slightly larger than the peak downward velocity. These variations are consistent with the well known velocity profile of ion beams inside the PSBL (Takahashi and Hones, 1988). The spatial/temporal pattern can thus locally lead to strong net Earthward and/or tailward energy flux. There is no one-to-one 


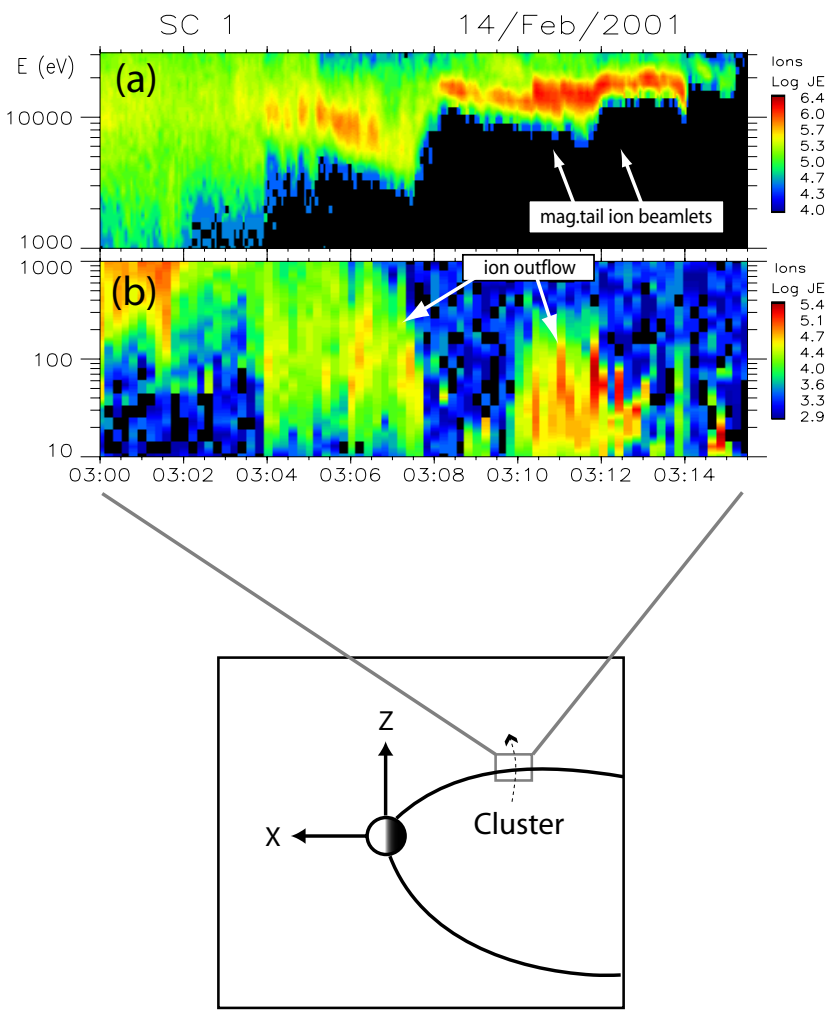

Fig. 10. Outbound crossing of the PSBL on 14 February 2001. (a) E-t spectrogram of ions (non-mass resolved) with $>1 \mathrm{keV}$. (b) E-t spectrogram of ions with $<1 \mathrm{keV}$.

correlation between enhanced downgoing kinetic energy flux of ions and upflowing kinetic energy flux of ion conics. However, since the energy flux of the magnetotail ions is overwhelmingly larger (1-2 orders of magnitude) than that of the upflowing ion conics, only small energy differences between downward and upward traveling ion beamlets are required to energize the ion conics. These small energy differences together with the temporal/spatial variations are probably impossible to identify in this energy flux comparison. Similarly, no one-to-one correlation between upflowing ion conics and either FAC or Alfvén waves exists. Thus, we conclude that this energy flux comparison does not provide any confirmation as to which of the three energy carriers was the actual driver but it shows that each of them carries enough energy flux to be the driver of ion conics. However, as argued above and also summarized in the discussion section, other reasons suggest that the ion beamlets were the driver.

The energy flux required to power weak auroral arcs, on the other hand, requires much more energy flux than was carried by the here reported ion outflow, and we will argue in Sect. 5.7 that the Alfvén waves were likely the driver.

\section{Second event: outbound crossing of the PSBL}

The importance of edges of magnetotail ion beamlets is further demonstrated with this second event. About $2.5 \mathrm{~h}$ after Cluster crossed the PSBL in the Southern Hemisphere, it crossed the opposite (northern) boundary of the PS at about the same radial distance and local time but this time during an outbound motion of the spacecraft (Fig. 10). Substorm recovery was also prevailing during this crossing as it was for the inbound event. At about 03:04 UT Cluster encountered energy-dispersed ions which lasted until 03:14 UT in the spacecraft frame while crossing the PSBL (Fig. 10a). These energetic ions were accompanied by intense ion outflow (Fig. 10b). In the following analysis, we will focus on the time period from about 03:08 to 03:14 UT (Fig. 11).

Figure 11 shows several magnetotail ion beamlets and associated phenomena. It is noted that most ion beamlets are not separated from one another which is different compared to the beamlet substructure reported for the inbound event (Sect. 3). One common signature between beamlets of both inbound and outbound events are the abrupt energy steps (or edges) (see arrows in Fig. 11a) of individual beamlets occurring from one sample interval (4s) to the next. Only the most apparent energy steps are marked with dashed lines and numbers but additional steps can be found in this interval. These steps separate individual beamlets from one another. In Keiling et al. (2004b), it was determined using multiple spacecraft measurements that the steps were caused by crossing spatial boundaries of flux tubes carrying the magnetotail ion beamlets (as opposed to a sudden appearance of a beamlet). The sharp boundaries have spatial scales of $<60 \mathrm{~km}$ since the ion fluxes changed abruptly from one sample to the next (4-s time resolution of the ion instrument) and the relative motion of spacecraft and ambient plasma was about $15 \mathrm{~km} / \mathrm{s}$. In comparison, the gyroradius of a $10-\mathrm{keV}$ ion is about $30 \mathrm{~km}$ at this location.

The most important observation in the context of this study is that these boundaries occurred simultaneously with other particle and field signatures. The boundaries numbered 1 through 5 are associated with simultaneously occurring enhanced ion outflows in comparison to their immediate surroundings, suggesting a causal relationship as was argued for the inbound event (Sect. 3). It is however also noted that ion outflow existed between the boundaries but with lesser intensities. The outflow shows pitch angles up to $90^{\circ}$, which is similar to the inbound event (note that the magnetic field direction is reversed compared to the inbound event, i.e., a pitch angle of $180^{\circ}$ corresponds to upward motion).

Electron acceleration coincided with the ion outflow region from 03:10 to 03:12 UT (Figs. 11d-f). Intense downflowing electrons were present as well as upflowing ones; both showing fine structure. A clear one-to-one relationship with all boundaries (labeled 1 through 5) was not apparent, although the strongest downgoing electron flow (white arrow in panel d) coincides with \#2. This lack of a clear 


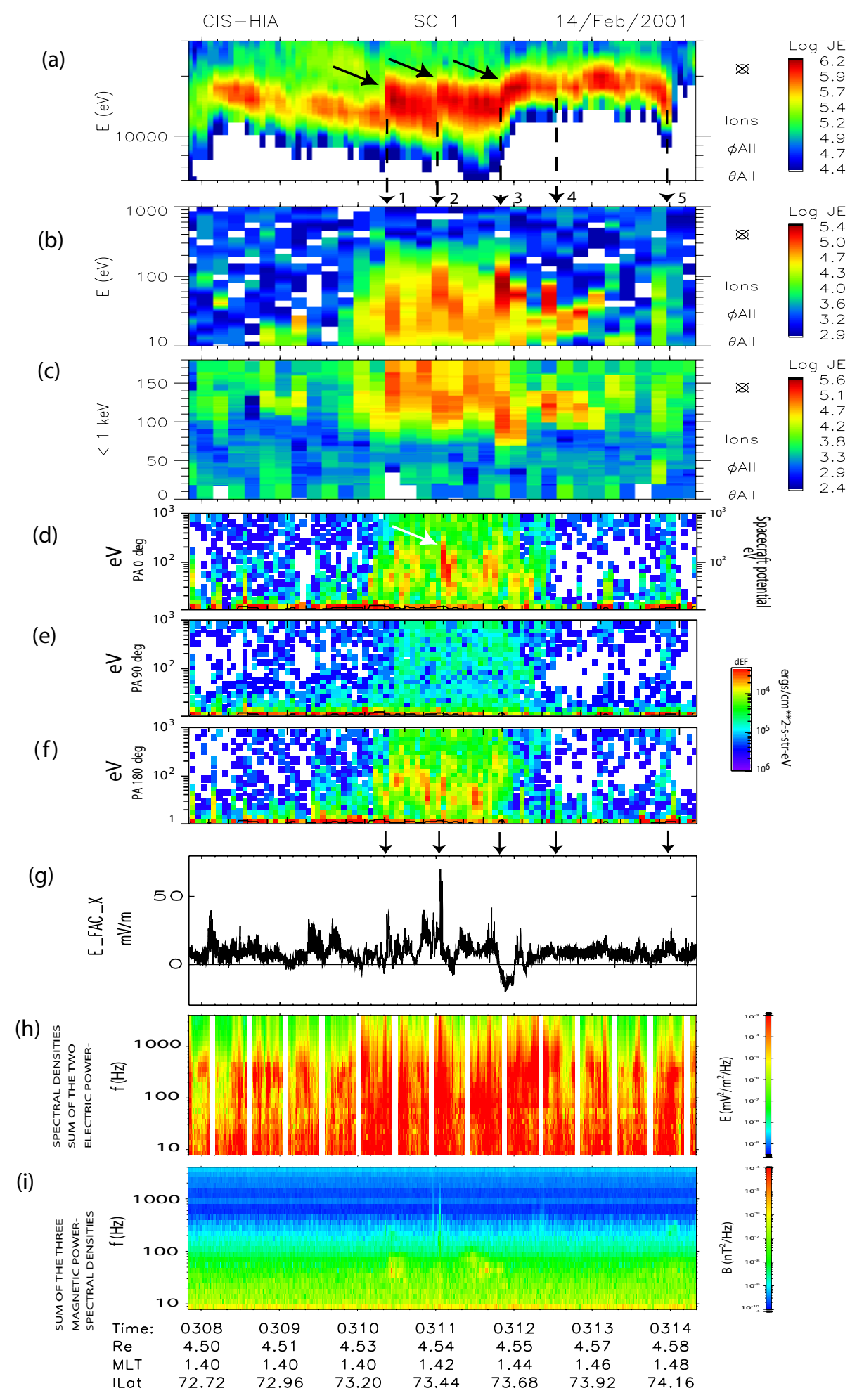

Fig. 11. Comparison of various quantities for the outbound crossing of the PSBL on 14 February 2001. (a) Ions with $>3 \mathrm{keV}$, (b) ions with $<1 \mathrm{keV}$, (c) pitch angle plot for ions with $<1 \mathrm{keV}$, (d-f) electrons for different pitch angle ranges, (g) $\mathrm{E}_{x}$ component of the electric field in field-aligned coordinates, (h, i) electric and magnetic field power spectral densities up to $4 \mathrm{kHz}$. 


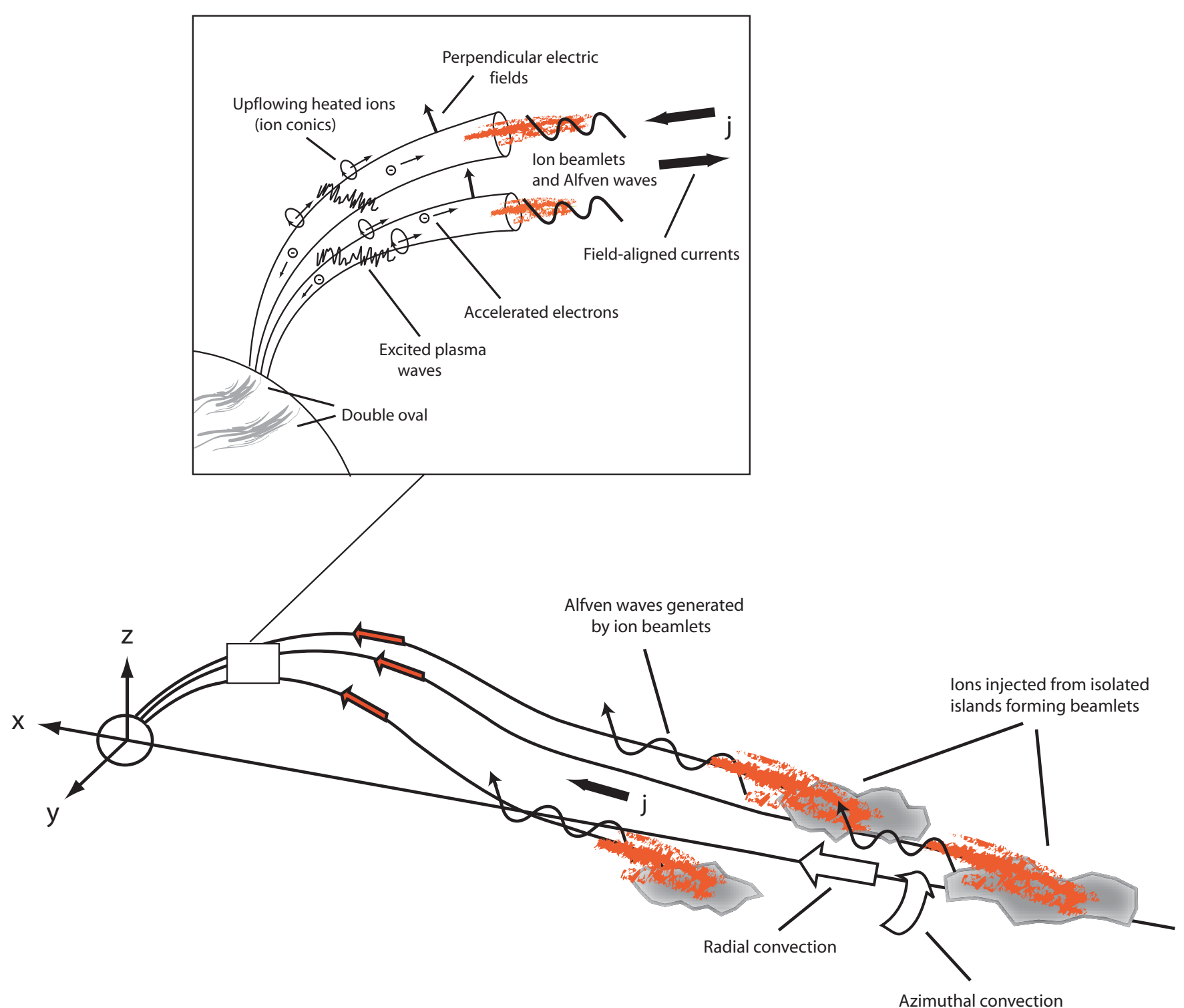

Fig. 12. Simplified cartoon showing the various phenomena that are possibly causally associated with the energy-dispersed ion beamlets observed during the PSBL crossings.

one-to-one relationship could be because the ion outflow was present during the entire time interval of electron flow (03:10-03:13:20 UT) without intermittent gaps (i.e., no ion outflow).

The perpendicular electric field, $E_{x}$, is shown in Fig. 11g. The arrows above this panel are placed at the same times as those above the first panel. In particular, the largest two spikes (arrows \#1 and \#2 and to a lesser degree \#3) line up well with the beamlet boundaries (Fig. 11a), giving further support to the scenario that these boundaries of the ion beamlets created perpendicular electric fields which in turn heated the upflowing ionospheric ions.

BEN and Alfvén waves were also present inside the beamlet carrying region, but simple and convincing one-to-one correlations as for the ion conics are not apparent; instead the waves cover the entire beamlet-carrying region as was the case for the inbound event. It is also noted that BEN was present deep inside the CPS with similar intensity as in the PSBL. The wave magnetic field data (Fig. 11i) showed isolated enhancements which approximately coincided with the beamlet boundaries. Thus, as for the inbound event, electromagnetic waves appear to be associated with these boundaries.

In conclusion, this outbound event supports the view that was developed for the inbound event, namely that the magnetotail ion beamlets, in particular their sharp edges (or boundaries) are driving other phenomena. 


\section{Discussion}

An important topic in M-I coupling is the impact of PSBL ion beams (or ion beamlets) on auroral dynamics (cf. Sect. 1). To investigate this topic, we presented two case studies showing various particle and field signatures in association with multiple ion beamlets that occurred during PSBL crossings at geocentric distance of about 4.5 Re. We compared the beamletcarrying region with neighboring regions to show that the signatures in the beamlet region were distinct from those of the other regions. On the basis of the particle and field signatures reported here, the following impacts (Fig. 12) of the ion beamlets are proposed and further discussed below:

- generation of ion conics

- generation of field-aligned electrons

- generation of broadband waves

- generation of electric field spikes

- generation of Alfvén waves

- association with FAC

- association with poleward arcs of double oval

We emphasize that our conclusions are based on the one-toone correlations of ion beamlets with the various particle and field signatures observed by Cluster 1 during the inbound PSBL crossing on 14 February 2001. Although the Cluster 3 observations during the same crossing also showed distinct particle and field signatures in various quantities inside the beamlet-carrying region, they did not show the clear one-toone correlations as observed in Cluster 1 data. However, we find it plausible that it is the exceptional separation of individual ion beamlets recorded by Cluster 1 that made it possible to see clear and convincing one-to-one correlations. Our findings were also supported by observations from a second event occurring during an outbound PSBL crossing which did not show well separated beamlets but showed well developed boundaries between beamlets similar to those recorded by Cluster 1 on the inbound PSBL crossing.

\subsection{Generation of ion conics}

Here we reported ion outflow in the form of bimodal ion conics at $4.5 \mathrm{Re}$ in a region which also carried the energetic ion beamlets from the magnetotail. A similar association of enhanced large pitch angle ion outflow and velocity-dispersed ions was recently reported in a Polar-spacecraft-based study at distances of $6 \mathrm{Re}$ and higher (Lennartsson, 2003). In addition, here we found that the enhanced conical outflow coincided with the edges (boundaries) of energetic magnetotail ion beamlets. These edges had perpendicular spatial scales of the order of gyroradii. Such boundaries were also reported in Lennartsson et al. (2001). The energy range of the conics was between 10 and $1000 \mathrm{eV}$, which is typical for ion conics ( $\mathrm{An}$ dre and Yau, 1997). It was inferred that the observed conics were heated at altitudes from 16000 to $22000 \mathrm{~km}$ which is within the range of previously observed ion conics (Kintner and Gorney, 1984; Peterson et al., 1992).

The free energy carried in ion beams is a potential source for the energization of ionospheric ion outflow. It has recently been suggested based on observations (using the Polar satellite) and simulations that ion shell distributions associated with PSBL ion flow excite Bernstein waves which in turn heat ion outflows to create the observed ion conics (Janhunen et al., 2003; Olsson et al., 2004). The simulated waves covered a frequency range of $50-500 \mathrm{~Hz}$. In an alternative scenario, also based on Polar observations and theoretical considerations (Lennartsson, 2003), perpendicular electric fields, generated by charge imbalances due to ion gyroradii differences between energetic magnetotail ions and electrons at density gradients of filamentary ion structures, are proposed to transversely accelerate outflowing ionospheric ions, thus creating ion conics. In turn, the acceleration and displacement of the ions are proposed to generate electric field turbulence. Thus, in this scenario, the ion conics cause the electric field turbulence whereas in Janhunen and Olsson's scenario, the electric field turbulence causes the heating of the ion conics.

Our observations can be compared with both scenarios. First, the ion beamlets reported here showed shell distributions in velocity space together with BEN (up to $4 \mathrm{kHz}$ ) and ion conics which is similar to the Janhunen and Olsson scenario (except the different frequency range). However, we found that the most intense and energetic ion conic outflows were found at or in the vicinity of beamlet edges (boundaries). Their scenario does not provide an explanation for the preferential edge location of the ion conics as was shown here. Furthermore, the edges often showed larger electric field spikes and ELF $(<12 \mathrm{~Hz})$ wave activity in the form of wave packets. These observations are consistent with the Lennartsson model (see also Sect. 5.4).

Either way, the clear and convincing one-to-one correlation between ion beamlets (or more precisely their edges) and ion conics strongly suggest that the beamlets provided the energy - which was shown to be sufficient - for the ion heating. We also rule out that Alfvén waves heated the outflowing ionospheric ions in a way described by Chaston et al. (2004) for ion heating below 1 Re altitude because at Cluster's location there was no indication of small-scale Alfvén waves with frequencies of $1 \mathrm{~Hz}$ or higher which are required for resonant heating of outflowing ions.

We showed that the ion conics were accelerated in the parallel field direction to produce bimodal ion conics as first reported by Klumpar et al. (1984). The current model by Lennartsson (2003) does not account for parallel electric fields. A plasma sheet mechanism first proposed by Alfvén and Fälthammar (1963) and later simulated by, for example, Schriver (1999) can generate upward directed electric 
fields due to the differences in mirror points of energetic PS ions and electrons. However, the simulation by Schriver et al. (1999) shows that most of the electric field is created at altitudes below the region from where our observed conics came. Nevertheless, a combination of the mechanisms proposed by Alfvén and Fälthammar (1963) and by Lennartsson (2003) might yield additional parallel acceleration of the ion conics. This possibility needs to be further investigated in future studies. On the other hand, the simulation by Janhunen et al. (2003), already mentioned above, produced field-aligned electric fields in addition to transverse electric fields in association with Bernstein modes. In their study it was argued that these fields accelerate electrons but it was not addressed whether they can contribute to parallel ion acceleration.

A constraining feature for the ion conic generation was that the conics showed no energy dispersion. It was inferred that the source regions of these conics was at times $6000 \mathrm{~km}$ below the spacecraft. Thus, the ions with the lowest $(\sim 30 \mathrm{eV})$ and highest $(1 \mathrm{keV})$ energies require at least $85 \mathrm{~s}$ and $14 \mathrm{~s}$, respectively, to reach the spacecraft. This would argue that the outflows lasted for at least $71 \mathrm{~s}(=85 \mathrm{~s}-14 \mathrm{~s})$. If the source region of the ion conics convected with the $\boldsymbol{E} \times \boldsymbol{B}$ drift during this time, no energy dispersion would occur as was observed. This convection would also cause an ion conic to convect in its entirety to a different $\mathrm{L}$ shell before it reached the spacecraft. Since we argued above that the edges of ion beamlets were associated with the heating of the ion conics, one might expect that the ion conics would be displaced from the edges due to this $\boldsymbol{E} \times \boldsymbol{B}$ drift which however was not observed. In Keiling et al. (2004b) it was concluded that the edges of ion beamlets on 14 February 2001 were caused by a distant tail source which itself convected with the $\boldsymbol{E} \times \boldsymbol{B}$ drift, thus creating a broad energy range on the same field line (see also Lennartsson et al., 2001). Therefore, the $\boldsymbol{E} \times \boldsymbol{B}$ drift of the source regions of both magnetotail beamlets and ion conics - both located on the same flux tubes could explain why no separation of beamlets and ion conics occurred.

In conclusion, in our opinion, the clear correlation of ion conics (and the electric field spikes, see Sect. 5.4) and the edges of magnetotail beamlets favors the Lennartsson (2003) model for the ion conic generation reported here.

\subsection{Generation of field-aligned electrons}

A distinct electron signature occurred in the beamletcarrying region. Electron flow with energies below $100 \mathrm{eV}$ was present throughout the region; but most importantly, the energy was raised to levels up to $1 \mathrm{keV}$ at times when enhanced ion conic outflows (or equivalently, at times when magnetotail ion beamlets) occurred. The electrons were field-aligned and anti-field-aligned with varying dominance in a particular direction; $90^{\circ}$ mirroring electrons were also observed at times. The downward and upward electron flow suggests that the electron acceleration process occurred both below and above the spacecraft. Moreover, the one-to-one correlation with the magnetotail ion beamlets again suggests that a process driven by the ion beamlets was causing the electron energization.

Electron acceleration in the auroral acceleration region by field-aligned potential drops is well known (e.g., Evans, 1974; Mozer et al., 1980). But it has also been shown that electrons are accelerated at much higher altitudes. Kinetic Alfvén waves have been associated with the acceleration of electrons in the field-aligned direction in both the auroral region and the PSBL (Chaston et al., 2000; Wygant et al., 2002). Further, Janhunen et al. (2003) showed in simulations that electrons can be accelerated in the field-aligned direction by Bernstein modes. Field-aligned and heated electrons in the PSBL have been broadly associated with the presence of ion conics and magnetotail ion beams although not necessarily in a causal relationship (e.g., Klumpar and Heikkila, 1985; Schriver et al., 1990).

Our observations favor a scenario in which the ion beamlets energetically drive the acceleration of the electrons because of the above-mentioned one-to-one correlation. This acceleration might be mediated via the generation of broadband waves. In particular, several studies showed correlations between density gradients and the presence of broadband waves (Marklund et al., 2001; Vaivads et al., 2003; Wahlund et al., 2003). Backrud et al. (2004) showed that these waves can have a component parallel to the ambient magnetic field. The scenario by Janhunen et al. (2003) (see previous paragraph) is consistent with these reports and thus a possible candidate for the electron acceleration observed here, although the authors did not associate the wave generation and the subsequent electron acceleration with the edges of ion beamlets. Kinetic Alfvén wave acceleration of electrons is not likely because the observed Alfvén waves did not show the small scales (frequencies of $1 \mathrm{~Hz}$ and higher) required to provide significant parallel electric fields at the location of 4-5 Re (Wygant et al., 2002).

Finally, it was peculiar that the electron energies were comparable to the ion conics' energies which will need to be explained in future studies.

\subsection{Generation of broadband waves}

Several broadband wave modes were recorded inside the beamlet-carrying region. First, broadband electrostatic noise (BEN) activity (up to $4 \mathrm{kHz}$ and possibly higher) was enhanced during the crossing of the beamlet-carrying region, although significant activity was also recorded deep into the CPS which was at times as strong as those in the PSBL. Second, electrostatic ELF waves $(<12.5 \mathrm{~Hz})$ were observed, sometimes in the form of wave packets. These wave packets appeared to be uncorrelated to the higher frequency BEN, but instead were often collocated with the boundaries of ion beamlets. ELF was also found inside beamlets that lasted 
for 1-2 min. Third, broadband electromagnetic waves were collocated with the boundaries of beamlets.

It has long been known that BEN is enhanced across the PSBL (Gurnett et al., 1976). However, it has proven to be very difficult to identify the wave modes and their driving source associated with BEN (e.g., Lakhina et al., 2000, and references therein). Many simulation studies exist showing different sources of BEN, but clear and unambiguous observational evidence does not exist because too many parameters are simultaneously observed. FAC (e.g., Ashour-Abdalla and Thorne, 1977), ion beams (e.g., Grabbe and Eastman, 1984), electron beams (e.g., Schriver and Ashour-Abdalla, 1989), and Alfvén waves (Backrud et al., 2004) have all been put forward as the driver of BEN. All these signatures were present during our events, making an identification more ambiguous.

BEN was enhanced during both downward and upward FAC inside the beamlet-carrying region, suggesting that the FAC were not the generator of BEN inside the beamletcarrying region. Janhunen et al. (2003) proposed that ion shell distributions similar to those reported here could excite several Bernstein modes in the frequency range $(50-500 \mathrm{~Hz})$. It was suggested by these authors that these waves could yield the signature of BEN. The lack of clear one-to-one correlations of the striated BEN and the beamlets, as reported here, might be due to the fact that BEN propagates obliquely to the ambient magnetic field.

Intense BEN was also present further into the CPS. If the ion beamlets did provide the energy for BEN, then it is also clear that other sources can drive BEN since in the CPS no ion beamlets were observed. In the CPS, upward FAC and inverted ion V's were present which are possible sources for those BEN.

In contrast, the here reported ELF wave packets $(10 \mathrm{~Hz})$ were often found at the boundaries of magnetotail ion beamlets. Such ELF turbulence can be explained with the electric field generation process as proposed by Lennartsson (2003) (see Sect. 5.1). Furthermore, the correlation of broadband electromagnetic waves and boundaries of ion beamlets suggests that the waves were energetically driven by the beamlets. The mechanism is as of yet unknown.

\subsection{Generation of electric field spikes}

Inside the broad region of electric field turbulence, larger electric field spikes were reported which often coincided with the edges of the magnetotail ion beamlets. No magnetic counterparts of the electric field spikes were present. Thus, we rule out that the spikes were of Alfvénic nature.

Instead, the generation of electric fields at beamlet edges is readily explained with the model by Lennartsson (2003) (see also Sect. 5.1). Lennartsson did not actually show a one-toone correlation of electric field spikes and individual beamlet edges, but it was shown that the turbulent electric field region coincided with the region of filamentary magnetotail ion flow at 6 Re and above. The filamentary ion structures showed gradients of the order of gyroradii. It was suggested that the generation of electric fields at the gradients and the subsequent acceleration of cold ions would lead to further electric field turbulence.

\subsection{Generation of Alfvén waves}

The region of beamlets was threaded by low-frequency Alfvén waves which showed both traveling and standing signatures. The Alfvén wave electric field amplitudes were of the order of $\mathrm{mV} / \mathrm{m}$ which is $1-2$ orders of magnitude smaller compared to Alfvén waves in the PSBL during times of substorm expansion phase (Wygant et al., 2000; Keiling et al., 2000). The event here occurred during the recovery phase. The signature of standing Alfvén waves is readily explained by the reflection of Alfvén waves off the ionosphere (Mallinckrodt and Carlson, 1978).

An association of Alfvén waves and ion flow in the lobePSBL region was recently proposed by Zelenyi et al. (2004). Dispersionless ion structures at $25 \mathrm{Re}$ were found on fluctuating magnetic field lines which were identified as Alfvénic. It was suggested that the ion structures injected in the distant magnetotail excite Alfvén waves on the same flux tube via the tail firehose instability. Although our ion beamlets show clear energy dispersion signatures, and thus are different from those reported in Zelenyi et al. (2004), it is also possible that our beamlets triggered a firehose instability which produced Alfvén waves. The condition for the firehose instability has to be met near the source region in the distant tail and cannot directly be verified for our event, but as argued in Zelenyi et al. (2004), this condition can be met in the far tail. Takada et al. (2005) also showed the simultaneous occurrence of Alfvén waves and ion flows in the magnetotail ( $>15 \mathrm{Re}$ ), and proposed that an ion cyclotron anisotropy instability could generate the Alfvén waves. In accordance with these two studies, it is thus possible that the Alfvén waves observed together with the beamlets by Cluster were generated by the beamlets in the far-tail region.

\subsection{Association with FAC}

The ion beamlet region carried FAC which carried significant Poynting flux towards the ionosphere. During the inbound event, both spacecraft first encountered downward FAC on entering the PBSL. The multiple ion beamlets were however not associated exclusively with one current direction. The ion beamlets of the first large-scale ion structure A (Figs. 2a and g) were located in the downward current region for both spacecraft and only secondary and tertiary structures (B and C) were located in the upward FAC region. Note that the secondary and tertiary ion structures were likely echoes of the first structure (Keiling et al., 2005a). Thus, it is possible that the downward currents are related to the generation of ion beamlets; additional investigations are required. 
The currents were associated with large-scale azimuthal convection at Cluster's location. This convection is possibly mediated via the FAC from the source region where azimuthal shear flow created this FAC. Such shear flow in the tail has been associated with bursty bulk flow (Angelopoulos et al., 2002). At this time, it remains open whether this shear flow is related to the beamlet injection mechanism.

\subsection{Association with poleward arcs of double oval}

As previously reported, both events occurred during a double auroral oval and the footprints of the beamlets mapped into the vicinity of the poleward arcs (Keiling et al., 2004b). The conjugacy of ion beamlets and auroral activity begs the question whether the ions were responsible for the auroral emissions. An association of VDIS with the double oval is consistent with the observation by Elphinstone et al. (1995). Other authors have also suggested that ion beams could drive auroral arcs (e.g., Marghitu et al., 2001; Janhunen et al., 2003; Olsson et al., 2004). For example, Olsson et al. (2004) suggested that the free energy contained in ion shell distributions observed in the PSBL plays a role in the energy transfer leading to stable auroral arcs.

The energy flux required to cause visible aurora ( $>1 \mathrm{erg} / \mathrm{cm}^{2} \mathrm{~s}$ at ionospheric altitude) is significantly larger (1-2 orders of magnitude) than the energy flux required to drive, for example, the ion conic heating. Although the ion beamlets carried such energy flux towards Earth (up to $4 \mathrm{erg} / \mathrm{cm}^{2} \mathrm{~s}$ when mapped to ionospheric altitudes using a mapping factor of 100), most of their energy flux however mirrored back, thus creating the ion shell distributions. Consistent flattening of these distributions was also not observed (Janhunen et al., 2003). Therefore, only relatively small amounts of beamlet energy were actually dissipated below the spacecraft. Instead, Alfvén waves were largely dissipated below the spacecraft showing a net Poynting flux toward the ionosphere of $>1 \mathrm{erg} / \mathrm{cm}^{2} \mathrm{~s}$ (mapped to ionospheric altitudes). This is above the threshold of visible aurora. Alfvén waves traveling in the PSBL have been associated with the driving of conjugate auroras (Wygant et al., 2000; Keiling et al., 2002). Keiling et al. (2002) showed a positive correlation of the Poynting flux of Alfvén waves and auroral luminosity. The Poynting flux reported here corresponds to the weaker auroras reported in Keiling et al. It is also noted that the FAC was downward on entering the PSBL, thus ruling the current out as a driver; at least in a way that is associated with upward FAC and auroral acceleration. We thus propose that the Alfvén waves generated by ion beams in the distant tail could drive the poleward arcs of the double oval. In this scenario, the ion beamlets are thus indirectly responsible for the auroral emissions.

\section{Conclusions}

It is well known that a rich phenomenology exists on PSBL field lines, but the causal relationship among these phenomena is not well established. For example, temporal or spatial correlations among phenomena are observed and theoretical work is provided to support a cause-effect relationship; however, several phenomena are often simultaneously present and the correlations are often only approximate, i.e., in the "close" temporal or spatial proximity, which makes an unambiguous identification of the cause-effect relationship difficult. Consequently, alternative scenarios are often proposed which can explain the same observations. The discrimination of competing scenarios is one of the challenges in magnetospheric physics.

Cluster observations presented here show that energydispersed ions in the PSBL energetically drive the heating of ion outflows forming ion conics, the field-aligned acceleration of electrons, and the generation of perpendicular electric fields and ELF turbulence. These conclusions were foremost based on convincing one-to-one correlations of the phenomena with the beamlet substructure of the dispersed ions. The beamlet substructure facilitated the identification of causeeffect relationships because it was possible to explain the fine structures of these associated phenomena with the beamlet substructure. An important signature was that the various correlations occurred at the boundaries (with narrow spatial scales of the order of a gyroradius) of individual ion beamlets. These boundaries "focused" the correlations to narrow regions which left less room for ambiguities. The association with beamlets was further supported by the abrupt changes that occurred at the boundaries to the regions adjacent to the beamlet-carrying region.

Additional support for our conclusions is that they are consistent with the observations and theoretical considerations of Lennartsson (2003) but also extend their observations. Lennartsson proposed a model where ion beam gradients with small perpendicular scale of the order of gyroradii create perpendicular electric fields which locally heat outflowing ions. This model accounts well for the observations of ion conics and electric field generation occurring at the boundaries of ion beamlets as reported here. Lennartsson, however, did not comment on plasma wave activity and parallel acceleration of both ions and electrons as reported here which would have allowed further comparisons of his observations to our event.

Furthermore, it was shown that the dispersed ions reported here could provide the free energy for BEN and electromagnetic broadband waves. The ions showed shell distributions in velocity space which have been proposed by Janhunen et al. (2003) and Olsson et al. (2004) to create BEN. Although convincing one-to-one correlations as shown for the ion conics, field-aligned electrons, and electric field spikes were not observed for BEN, it is very probable that the BEN was energetically driven by the ion beamlets because other scenarios 
were found to be less likely, and the perpendicular propagation of BEN might have obscured this one-to-one correlation. In contrast, electromagnetic broadband waves showed a oneto-one correlation with the ion beamlets.

In addition, we showed new observations regarding the source region of the magnetotail ion beamlets. In Keiling et al. (2004b), an injection scenario was proposed for the highly structured multiple dispersed ion beams seen in the events reported here. Two new observations add to their scenario, namely (1) that the source region of the magnetotail ion beams generated a downward FAC which connected azimuthal shear flow in the far tail with the ionosphere, and (2) that both traveling and standing Alfvén waves accompanied the ion beamlets. We proposed that the Alfvén waves were possibly generated by the beamlets themselves in the distant tail via ion beam-related instabilities (Zelenyi et al., 2004; Takada et al., 2005). Additional event studies are required to determine whether these observations are common.

This work can also be viewed in the framework of how the energy coming from the magnetotail is distributed among various energy carriers. Both theoretical and observational works show that FAC, ion beams, and Alfvén waves contribute to this energy transfer (e.g., Schriver et al., 2003). Here we showed that the FAC carried the largest amount of energy flux (almost one order of magnitude larger than for the other two energy carriers) on 14 February 2001. This energy flux however was not dissipated to create the phenomena discussed in this report but must have been dissipated at much lower altitude possibly the ionosphere (for example, via Joule heating). Alfvén waves and ion beamlets carried comparable amounts of energy flux. Much of the Alfvénic energy flux was dissipated below the spacecraft; perhaps into auroral emissions as argued here. In contrast, most of the ion beamlet energy flux was mirrored at lower altitude and returned to the magnetotail (which was manifested in the shell distribution). Such mirroring ion beamlets can lead to bouncing ion clusters inside the PSBL (Ashour-Abdalla et al., 1993; Keiling et al., 2005a) and thus can again drive the same processes - as described here - on their return. It was shown that only small amounts of energy flux are required to drive the locally observed ion outflow, and thus most of the beamlet energy is eventually thermalized in the CPS. Thus, we conclude that Alfvén waves and FAC contributed more energy flux towards M-I coupling for this particular event which might be typical for the recovery phase - compared to magnetotail ion beamlets. Nevertheless, many phenomena were driven by these ion beamlets in regions above the auroral acceleration region. Furthermore, if the Alfvén waves were indeed generated by ion beamlets in the distant magnetotail, then this shows that ion beamlets have an impact on magnetotail dynamics not only at Cluster's location and below but already starting at the source region.

Acknowledgements. This work was supported by NASA grant NNG04GF23G. We thank F. Mozer for providing Cluster electric field (EFW) data, and A. Viljanen from the Finnish Meteorological Institute for the IMAGE ground magnetometer data. We also thank the reviewers for their critical comments.

Topical Editor I. A. Daglis thanks W. K. Peterson and M. Hirahara for their help in evaluating this paper.

\section{References}

Alfvén, H. and Fälthammar, C. G.: Cosmical Electrodynamics, Clarendon, Oxford, England, 1963.

Andre, M. and Yau, A.: Theories and observations of ion energization and outflow in the high latitude magnetosphere, Space Sci. Rev., 80, 27-48, 1997.

Angelopoulos, V., Chapman, J. A., Mozer, F. S., et al.: Plasma sheet electromagnetic power generation and its dissipation along auroral field lines, J. Geophys. Res., 107, A8, doi:10.1029/2001JA900136, 2002.

Ashour-Abdalla, M. and Thorne, R. M.: The importance of electrostatic ion-cyclotron instability for quiet-time proton auroral precipitation, Geophys. Res. Lett., 4, 45-48, 1977.

Ashour-Abdalla, M., Zelenyi, L. M., Bosqued, J. M., and Kovrazhkin, R. A.: Precipitation of fast ion beams from the plasma sheet boundary layer, Geophys. Res. Lett., 19, 617-620, 1992.

Ashour-Abdalla, M., Bosqued, J. M., El-Alaoui, M., et al.: A stochastic sea: The source of plasma sheet boundary layer ion structures observed by Cluster, J. Geophys. Res., 110, A12, doi:10.1029/2005JA011183, 2005.

Backrud, M., André, M., Balogh, A., et al.: Identification of broadband waves above the auroral acceleration region: Cluster observations, Ann. Geophys., 22, 4203-4216, 2004, http://www.ann-geophys.net/22/4203/2004/.

Bosqued, J. M., Ashour-Abdalla, M., El Alaoui, M., et al.: Dispersed ion structures at the poleward edge of the auroral oval: Low-altitude observations and numerical modeling, J. Geophys. Res., 98, 19 181-19204, 1993.

Carlson, C. W., McFadden, J. P., Ergun, R. E., et al.: FAST observations in the downward auroral current region: Energetic upgoing electron beams, parallel potential drops, and ion heating, Geophys. Res. Lett., 25, 2017-2020, 1998.

Cattell, C.: Association of field-aligned currents with small-scale auroral phenomena, Geophys. Monograph, 28, 304-314, 1984.

Chaston, C. C., Carlson, C. W., Ergun, R. E., and McFadden, J. P.: Alfvén Waves, Density Cavities and Electron Acceleration Observed from the FAST Spacecraft, Physica Scripta, T84, 64-68, 2000.

Chaston, C. C., Bonnell, J. W., Carlson, C. W., et al.: Auroral ion acceleration in dispersive Alfvén waves, J. Geophys. Res., 109, A4, doi:10.1029/2003JA010053, 2004.

Dubinin, E. M., Israelevich, P. L., Nikolaeva, N. S., et al.: Auroral electromagnetic disturbances at an altitude of $900 \mathrm{~km}$ : The relationship between the electric and magnetic field variations, Planet. Space Sci., 38, 97-108, 1990.

Eastman, T. E., Frank, L. A., Peterson, W. K., and Lennartsson, W.: The plasma sheet boundary layer, J. Geophys. Res., 89, 15531572, 1984.

Elphinstone, R.D., Hearn, D. J., Cogger, L. L., et al.: The double oval UV auroral distribution: 2 . The most poleward arc sys- 
tem and the dynamics of the magnetotail, J. Geophys. Res., 100, 12 093-12 102, 1995.

Escoubet, C. P., Schmidt, R., and Goldstein, M. L.: Cluster - Science and Mission Overview, Space Sci. Rev., 79(1/2), 11-32, 1997.

Evans, D. S.: Precipitation electron flux formed by a magnetic-fieldaligned potential difference, J. Geophys. Res., 79, 2853-2863, 1974.

Forbes, T., Hones, E. W., Bame, S. J., et al.: Evidence for the tailward retreat of a magnetic neutral line in the magnetotail during substorm recovery, Geophys. Res. Lett., 8, 261-264, 1981.

Grabbe, C. L. and Eastman, T. E.: Generation of broadband electrostatic noise by ion beam instabilities in the magnetotail, J. Geophys. Res., 89, 3865-3872, 1984.

Grigorenko, E. E., Fedorov, A., and Zelenyi, L. M.: Statistical study of transient plasma structures in magnetotail lobes and plasma sheet boundary layer: Interball-1 observations, Ann. Geophys., 20, 329-340, 2002,

Gurnett, D. A., Frank, L. A., Lepping, R. P., et al.: Plasma waves in the distant magnetotail, J. Geophys. Res., 81, 6059-6071, 1976.

Hasegawa, A.: Beam production at plasma boundaries by kinetic Alfvén waves, J. Geophys. Res., 92, 11 221-11 223, 1987.

Hirahara, M., Yamazaki, A., Seki, K., et al.: Characteristics of downward flowing ion energy dispersions observed in the lowaltitude central plasma sheet by Akebono and DMSP, J. Geophys. Res., 102, 4821-4840, 1997.

Hirahara, M., Horwitz, J. L., Moore, T. E., et al.: Relationship of topside ionospheric ion outflows to auroral forms and precipitation, plasma waves, and convection observed by Polar, J. Geophys. Res., 103, 17 391-17 410, 1998.

Hultqvist, B., Lundin, R., Stasiewicz, K., et al.: Simultaneous observations of upward moving field-aligned energetic electrons and ions on auroral zone field lines, J. Geophys. Res., 93, 97659776, 1988.

Hultqvist, B.: On the acceleration of positive ions by high-altitude, large-amplitude electric filed fluctuations, J. Geophys. Res., 101, 27 111-27 122, 1996.

Janhunen, P., Olsson, A., Vaivads, A., and Peterson, W. K.: Generation of Bernstein waves by ion shell distributions in the auroral region, Ann. Geophys., 21, 1-11, $2003 \mathrm{a}$.

Kan, J. R. and Akasofu, S.-J.: Energy source and mechanisms for accelerating the electrons and driving the field-aligned currents of the discrete auroral arc, J. Geophys. Res., 81, 5123-5130, 1976.

Kazama, Y. and Mukai, M.: Multiple energy-dispersed ion signatures in the near-Earth magnetotail: Geotail observation, Geophys. Res. Lett., 30, A7, doi:10.1029/2002GL016637, 2003.

Kazama, Y. and Mukai, M.: Simultaneous appearance of energydispersed ion signatures of ionospheric and magnetotail origins in the near-Earth plasma sheet, J. Geophys. Res., 110, A7, doi:10.1029/2004JA010820, 2005.

Keiling, A., Wygant, J. R., Cattell, C., et al.: Large Alfvén wave power in the plasma sheet boundary layer during the expansion phase of substorms, Geophys. Res. Lett., 27, 3169-3172, 2000.

Keiling, A., Wygant, J. R., Cattell, C., et al.: Correlation of Alfvén wave Poynting flux in the plasma sheet at $4-7 \mathrm{R}_{E}$ with ionospheric electron energy flux, J. Geophys. Res., 107, A7, doi:10.1029/2001JA900140, 2002.

Keiling, A., Rème, H., Dandouras, I., et al.: New proper- ties of energy-dispersed ions in the plasma sheet boundary layer observed by Cluster, J. Geophys. Res., 109, A5, doi:10.1029/2003JA010277, 2004a.

Keiling, A., Rème, H., Dandouras, I., et al.: Transient ion beamlet injections into spatially separated PSBL flux tubes observed by Cluster-CIS, Geophys. Res. Lett., 31, A12, doi:10.1029/2004GL020192, 2004b.

Keiling, A., Parks, G. K., Rème, H., et al.: Bouncing ion clusters in the plasma sheet boundary layer observed by Cluster-CIS, J. Geophys. Res., 110, A9, doi:10.1029/2004JA010497, 2005a.

Keiling, A., Parks, G. K., Wygant, J. R., et al.: Some properties of Alfvén waves: Observations in the tail lobes and the plasma sheet boundary layer, J. Geophys. Res., 110, A10, doi:10.1029/2004JA010907, 2005b.

Kintner, P. M. and Gorney, D. I.: A search for the plasma processes associated with perpendicular ion heating, J. Geophys. Res., 89, 937-944, 1984.

Klumpar, D. M., Peterson, W. K., and Shelley, E. G.: Direct evidence for two-stage (bimodal) acceleration of ionospheric ions, J. Geophys. Res., 89, 10 779-10787, 1984.

Lakhina, G. S., Tsurutani, B. T., Kojima, H., and Matsumoto, H.: Broadband plasma waves in the boundary layers, J. Geophys. Res., 105, 27 791-27 832, 2000.

Lennartsson, O. W., Trattner, K. J., Collin, H. L., and Peterson, W. K.: Polar/Toroidal Imaging Mass-Angle Spectrograph survey of earthward field-aligned proton flows from the nearmidnight tail, J. Geophys. Res., 106, 5859-5871, 2001.

Lennartsson, O. W.: In situ Polar observations of transverse coldion acceleration: Evidence that electric field generation is a hot ion finite gyroradii effect, J. Geophys. Res., 108, A4, doi:10.1029/2002JA009663, 2003.

Lui, A. T. Y., Frank, L. A., Ackerson, K. L., et al.: Plasma flows and magnetic field vectors in the plasma sheet during substorms, J. Geophys. Res., 83, 3849-3858, 1978.

Lyons, L. R. and Speiser, T. W.: Evidence for current sheet acceleration in the geomagnetic tail, J. Geophys. Res., 87, 2276-2286, 1982.

Lyons, L. R. and Evans, D. S.: An association between discrete aurora and energetic particle boundaries, J. Geophys. Res., 89, 2395-2400, 1984

Mallinckrodt, A. J. and Carlson, C. W.: Relations between transverse electric fields and field-aligned currents, J. Geophys. Res., 83, 1426-1432, 1978.

Marghitu, O.: Observational evidence for a potential relationship between visible auroral arcs and ion beams - a case study, Phys. Chem. Earth, 26, 223-228, 2001.

Mozer, F. S., Cattell, C. A., Hudson, M. K., et al.: Satellite measurements and theories of low altitude auroral particle acceleration, Space Sci. Rev., 27, 155-213, 1980.

Olsson, A., Janhunen, P., and Peterson, W.: Ion shell distributions as free energy source for plasma waves on auroral field lines mapping to plasma sheet boundary layer, Ann. Geophys., 22, 21152133, 2004, http://www.ann-geophys.net/22/2115/2004/.

Parks, G. K., McCarthy, M., Fitzenreiter, R. J., et al.: Particle and field characteristics of the high-latitude plasma sheet boundary layer, J. Geophys. Res., 89, 8885-8906, 1984.

Parks, G. K., Chen, L. J., McCarthy, M., et al.: New observations of ion beams in the plasma sheet boundary layer, Geophys. Res. 
Lett., 25, 3285-3288, 1998.

Peterson, W. K., Collin, H. L., Doherty, M. F., and Bjorklund, C. M.: $\mathrm{O}(+)$ and $\mathrm{He}(+)$ restricted and extended (bi-modal) ion conic distributions, Geophys. Res. Lett., 19, 1439-1442, 1992.

Reiff, P., Collin, H. L., Craven, J. D., et al.: Determination of auroral electrostatic potentials using high- and low-altitude particle distributions, J. Geophys. Res., 93, 7441-7465, 1988.

Sauvaud, J.-A., Popescu, D., Delcourt, D. C., et al.: Sporadic plasma sheet ion injections into the high-altitude auroral bulge: Satellite observations, J. Geophys. Res., 104, 28 565-28 577, 1999.

Sauvaud, J.-A. and Kovrazhkin, R. A.: Two types of energydispersed ion structures at the plasma sheet boundary, J. Geophys. Res., 109, A12, doi:10.1029/2003JA010333, 2004.

Schindler, K. and Birn, J.: On the generation of field-aligned plasma flow at the boundary of the plasma sheet, J. Geophys. Res., 92, 95-107, 1987.

Schriver, D. and Ashour-Abdalla, M.: Broadband electrostatic noise due to field-aligned currents, Geophys. Res. Lett., 16, 899-902, 1989.

Schriver, D., Ashour-Abdalla, M., Treumann, R., et al.: The lobe to plasma sheet boundary layer transition - Theory and observations, Geophys. Res. Lett., 17, 2027-2030, 1990.

Schriver, D.: Particle simulation of the auroral zone showing parallel electric fields, waves, and plasma acceleration, J. Geophys. Res., 104, 14 655-14 670, 1999.

Schriver, D., Ashour-Abdalla, M., Strangeway, R. J., et al.: FAST/Polar conjunction study of field-aligned auroral acceleration and corresponding magnetotail drivers, J. Geophys. Res., 108, A9, doi:10.1029/2002JA009426, 2003.

Sergeev, V. A., Sauvaud, J.-A., Popescu, D., et al.: Plasma sheet ion injections into the auroral bulge: Correlative study of spacecraft and ground observations, J. Geophys. Res., 105, 18 465-18481, 2000.

Sergeev, V. A., Sauvaud, J.-A., Rème, H., et al.: Sharp boundary between the inner magnetosphere and active outer plasma sheet, Geophys. Res. Lett., 30, A15, doi:10.1029/2003GL017095, 2003.
Strangeway, R. J., Ergun, R. E., Su, Y.-J., et al.: Factors controlling ionospheric outflows as observed at intermediate altitudes, J. Geophys. Res., 110, A3, doi:10.1029/2004JA010829, 2005.

Takada, T., Seki, K., Hirahara, M., et al.: Statistical properties of low-frequency waves and ion beams in the plasma sheet boundary layer: Geotail observations, J. Geophys. Res., 110, A2, doi:10.1029/2004JA010395, 2005.

Takahashi, K. and Hones Jr., E. W.: ISEE 1 and 2 observations of ion distributions at the plasma sheet-tail lobe boundary, J. Geophys. Res., 93, 8558-8582, 1988.

Wygant, J. R., Keiling, A., Cattell, C. A., et al.: Polar-spacecraft based comparison of intense electric fields and Poynting flux near and within the plasma sheet-tail lobe boundary to UVI images: An energy source for the aurora, J. Geophys. Res., 105, 18675$18692,2000$.

Wygant, J. R., Keiling, A., Cattell, C. A., et al.: Evidence for kinetic Alfvén waves and parallel electron energization at 5-7 RE altitudes in the plasma sheet boundary layer, J. Geophys. Res. 107, A8, doi:10.1029/2001JA900113, 2002.

Wygant, J. R., Cattell, C. A., Lysak, R., et al.: Cluster observations of an intense normal component of the electric field at a thin reconnecting current sheet in the tail and its role in the shock-like acceleration of the ion fluid into the separatrix region, J. Geophys. Res., 110, A9, doi:10.1029/2004JA010708, 2005.

Yau, A. and Andre, M.: Sources of ion outflow in the high latitude ionosphere, Space Sci. Rev., 80, 1-25, 1997.

Zelenyi, L. M., Kovrazkhin, R. A., and Bosqued, J. M.: Velocitydispersed ion beams in the nightside auroral zone - AUREOL 3 observations, J. Geophys. Res., 95, 12 119-12 139, 1990.

Zelenyi, L. M., Grigorenko, E. E., and Fedorov, A. O.: Spatialtemporal ion structures in the Earth's magnetotail: Beamlets as a result of nonadiabatic impulse acceleration of the plasma, JETP Lett., 80, 663-673, 2004. 